\title{
The Evolution of Price Competition Game on Complex Networks
}

\author{
Feng Jie Xie $\mathbb{I}^{1,2}$ and Jing Shi $\mathbb{D}^{3}$ \\ ${ }^{1}$ School of Modern Posts, Xi'an University of Posts and Telecommunications, Xi'an, ShaanXi, China \\ ${ }^{2}$ Industrial Economics Research Institute, Xi'an University of Posts and Telecommunications, Xi'an, ShaanXi, China \\ ${ }^{3}$ Department of Mechanical and Materials Engineering, College of Engineering \& Applied Science, University of Cincinnati, \\ Cincinnati, $\mathrm{OH}$, USA \\ Correspondence should be addressed to Jing Shi; jing.shi@uc.edu
}

Received 29 November 2017; Revised 22 April 2018; Accepted 12 May 2018; Published 9 July 2018

Academic Editor: Shahadat Uddin

Copyright (C) 2018 Feng Jie Xie and Jing Shi. This is an open access article distributed under the Creative Commons Attribution License, which permits unrestricted use, distribution, and reproduction in any medium, provided the original work is properly cited.

\begin{abstract}
The well-known "Bertrand paradox" describes a price competition game in which two competing firms reach an outcome where both charge a price equal to the marginal cost. The fact that the Bertrand paradox often goes against empirical evidences has intrigued many researchers. In this work, we study the game from a new theoretical perspective-an evolutionary game on complex networks. Three classic network models, square lattice, WS small-world network, and BA scale-free network, are used to describe the competitive relations among the firms which are bounded rational. The analysis result shows that full price keeping is one of the evolutionary equilibriums in a well-mixed interaction situation. Detailed experiment results indicate that the price-keeping phenomenon emerges in a square lattice, small-world network and scale-free network much more frequently than in a complete network which represents the well-mixed interaction situation. While the square lattice has little advantage in achieving full price keeping, the small-world network and the scale-free network exhibit a stronger capability in full price keeping than the complete network. This means that a complex competitive relation is a crucial factor for maintaining the price in the real world. Moreover, competition scale, original price, degree of cutting price, and demand sensitivity to price show a significant influence on price evolution on a complex network. The payoff scheme, which describes how each firm's payoff is calculated in each round game, only influences the price evolution on the scale-free network. These results provide new and important insights for understanding price competition in the real world.
\end{abstract}

\section{Introduction}

The well-known "Bertrand paradox" describes a game situation in which two firms engage in price competition in a static setting [1]. They sell a homogeneous good and have identical unit costs. The outcome is that both charge a price equal to the marginal cost. The game is called a paradox because the two firms could easily earn positive profits by charging a higher price. It has been studied by many researchers, indicating that some reasonable modifications to the Bertrand setting indeed resolve the paradox. Firstly, within the homogeneous-good framework, the Bertrand paradox can be resolved by introducing capacity constraints [2], sluggish consumers [3], the endogenous choice of production technologies [4], or the endogenous timing of price decisions [5]. Furthermore, differentiation $[6,7]$, uncertainty with respect to demand $[8,9]$ or product quality $[10,11]$, and nonconstant unit cost [12-15] are found to be the important factors for eliminating the Bertrand paradox. Thirdly, many works in laboratory settings focus on the oligopoly competition but do not observe the Bertrand equilibrium. This can be mainly attributed to the relaxation of the assumption regarding the rationality of economic agents in the laboratory environment [16-18].

Recently, the studies about the evolutionary game on complex networks reveal that topological structures can directly influence the evolution equilibrium of the game. In a pioneer work, Nowak and May introduced a twodimensional spatial lattice, that is, a square lattice, to analyze the evolution of a prisoner's dilemma game (PDG) [19]. They observed a remarkable cooperation level in this noncooperative game. Following this work, a wealth of studies provides 
additional evidence for the positive effect of various spatial lattices on cooperation in PDG [20-24]. Starting from the discovery of the small-world network $[25,26]$ and scalefree network [27], many studies about the evolution of PDG on these two network styles have emerged [28-32]. Furthermore, other game models, such as the snowdrift game, public good game, and stag hunt game, have been introduced to characterize different game situations among players. The studies about the evolution of these games on the small-world network and scale-free network yield abundant outcomes [33-38].

Inspired by the above works, we attempt to explore the Bertrand game from a new theoretical perspective-an evolutionary game on complex networks. The justification of such a perspective lies in two aspects. First, firms in the real world are bounded rational, but not complete rational. Huck et al. provided experimental tests for various learning theories in Bertrand games and concluded that firms imitate the most successful behavior $[39,40]$. This is evidence of the bounded rationality of firms facing a price decision in the real world. Second, the previous works find that competitive relations of firms in the real world have typical structural properties of complex networks [41-43]. Integrating the two aspects, we confirm that the evolutionary game theory on complex networks is suitable to study the price competition problem of bounded rational firms which have complex competitive relations.

To this end, some modifications to the Bertrand model are inevitable. Firstly, the evolutionary game theory studies the strategy evolution of large populations who are bounded rational $[44,45]$. Then, "two complete rational players" in a static setting should be modified as "a lot of bounded rational players" in an evolutionary dynamic setting. Furthermore, the interactions between players are supposed to happen at random in the evolutionary game theory, that is, the players interact in a well-mixed situation $[44,45]$. Also, while the complex network is introduced, the interaction of firms should be based on the complex competitive relations. We study price evolution under these new modifications. This is the first work that understands the price competition problem with the consideration of competition relations among the firms who charge the price. Our study explores the emergence of price keeping and identifies the positive effect of a complex competition relation on price keeping. Some important economic factors which influence the price evolution are also found and analyzed.

The remainder of this paper is organized as follows. Section 2 describes the model. Section 3 presents the theoretical analysis. Section 4 provides the simulation results and explanations. Section 5 summarizes our findings and concludes the paper.

\section{The Evolution of the Price Competition Game on Complex Networks}

As the first attempt of exploring the price competition problem from the theoretical perspective of the evolutionary game on complex networks, three widely applied network models are used to characterize the competitive interactions between firms, namely, square lattice [19], WS small-world network $[25,26]$, and BA scale-free network [27]. The average connectivity among WS networks and BA networks is set as four which is identical to that of a square lattice. Each node in networks is occupied by a firm. Each edge defines a competitive relation between two firms.

Based on the Bertrand model, the payoff $\pi_{i}\left(p_{i}, p_{j}\right)$ of firm $i$ who has a competitive relation with firm $j$ depends on their prices $p_{i}$ and $p_{j}$. That is

$$
\pi_{i}\left(p_{i}, p_{j}\right)= \begin{cases}\frac{\left(p_{i}-C\right) \cdot D\left(p_{i}\right)}{2}, & \text { if } p_{i}=p_{j}, \\ \left(p_{i}-C\right) \cdot D\left(p_{i}\right), & \text { if } p_{i}<p_{j}, \\ 0, \quad \text { if } p_{i}>p_{j}, & \end{cases}
$$

where $C$ is the constant unit cost and $D\left(p_{i}\right)$ is the demand function which generally has an expression $D\left(p_{i}\right)=a-u \cdot p_{i}$. In the expression, $a$ is the maximum of demand quantity while $p_{i}=0$ and $u(u>0)$ are the slope of demand function which indicates the sensitivity of demand to price. Without losing the generality, we set the constant unit cost as $C=0$ such that the model is simplified. Accordingly, the payoff $\pi_{i}\left(p_{i}, p_{j}\right)$ becomes

$$
\pi_{i}\left(p_{i}, p_{j}\right)= \begin{cases}\frac{p_{i} \cdot\left(a-u \cdot p_{i}\right)}{2}, & \text { if } p_{i}=p_{j}, \\ p_{i} \cdot\left(a-u \cdot p_{i}\right), & \text { if } p_{i}<p_{j}, \\ 0, \quad \text { if } p_{i}>p_{j} . & \end{cases}
$$

Under the framework of the evolutionary game theory, firms are bounded rational and thus have no capability to make the perfect decision of setting the price at marginal cost. They just make a simple decision: keep the original price $p(p>0)$ or cut the price to $p^{*}\left(p^{*}=\lambda p, 1>\lambda>0\right)$, where $\lambda$ is the degree of price cutting.

All firms simultaneously decide what prices they should offer. Each firm uses the same price for all of its competitive relations, that is, for all of its neighbor firms. The payoff of a firm can be measured by two payoff schemes: accumulated payoff or average payoff. It is worth mentioning that under different payoff schemes, the effects of scale-free networks on cooperation are different accordingly [31, 46-48]. Therefore, the payoff scheme is one of the important factors to be examined in the current work.

Price evolution is carried out implementing the rule of imitate best. In the previous studies of the evolutionary game on complex networks, various imitation rules are provided, such as imitate best $[46,49-51]$, imitate better with probability $[46,52-54]$, and imitate better with probability and error $[46,55,56]$. Here, we use the rule of imitate best, because the work of Huck et al. provides experimental evidence that firms imitate the most successful behavior, that is, the best one $[39,40]$.

The above price competition game and evolution mechanism can be described more specifically as follows. In each round of the game, that is, at each game time $t$, each firm offers a price $p_{i, t}$. The payoff of a firm at game time $t$ is 
TABLe 1: Order of $R, S, T$, and $P$ and the corresponding evolutionary equilibrium.

\begin{tabular}{lccc}
\hline Range of $\lambda$ & Range of $\beta$ & Order of $R, S, T$, and $P$ & Evolutionary equilibrium \\
\hline \multirow{2}{*}{$1>\lambda \geq 0.5$} & $\beta>1+\lambda$ & $T>R>P>S$ & Full price cutting \\
& $1+\lambda>\beta>1$ & $T>P>R>S$ & Full price cutting \\
$0.5>\lambda>0$ & $1+\lambda>\beta>1$ & $T>P>R>S$ & Full price cutting \\
& $\left(1-2 \lambda^{2}\right) /(1-2 \lambda)>\beta>1+\lambda$ & $T>R>P>S$ & Full price cutting \\
\hline & $\beta>1-2 \lambda^{2} / 1-2 \lambda$ & $R>T>P>S$ & Full price cutting or full price keeping \\
\hline
\end{tabular}

calculated by accumulated payoff or average payoff. Under the accumulated payoff scheme, the payoff $U_{i, t}$ of firm $i$ is the sum over all interactions of its neighbor firms, and can be written as $U_{i, t}=\sum_{j \in \Omega_{i}} \pi_{i, t}\left(p_{i, t} p_{j, t}\right)$, where $\Omega_{i}$ is the set of neighbor firms of $i$. Under the average payoff scheme, the payoff $U_{i, t}$ of firm $i$ is obtained by dividing the accumulated payoff by the number $n_{\Omega_{i}}$ of its neighbor firms, that is, $U_{i, t}=\sum_{j \in \Omega_{i}} \pi_{i, t}\left(p_{i, t,} p_{j, t}\right) / n_{\Omega_{i}}$. After all firms obtain payoffs in game time $t$, they update prices simultaneously. When firm $i$ updates its price, it compares the payoffs between itself and all of its neighbor firms and adopts the price that yields the highest payoff in game time $t$. After all firms have updated their prices, the next game time $t+1$ begins.

\section{Analysis}

According to payoff function (2), for each pair of firms who have a competitive relation, they both receive $p(a-u p) / 2$ upon mutual price $p$, or $p^{*}\left(a-u \cdot p^{*}\right) / 2$ upon mutual price $p^{*}$. One offering price $p^{*}$ receives an amount $p^{*}\left(a-u p^{*}\right)$ while the other offering price $p$ receives 0 payoff. Such a price-competitive situation can be described by payoff matrix $M_{1}$. That is

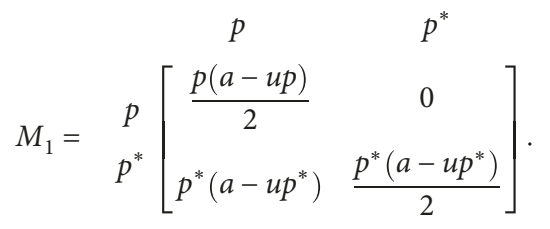

According to the evolutionary game theory, the relative order of four elements of payoff matrix $M_{1}$ can lead to different evolutionary equilibriums $[57,58]$. Denoting four payoffs in the matrix $M_{1}$, respectively, as $R=p(a-u p) / 2, T=p^{*}(a$ $\left.-u p^{*}\right), P=p^{*}\left(a-u \cdot p^{*}\right) / 2$, and $S=0$, the relative order of matrix elements $R, S, T$, and $P$ is analyzed in the following:

(1) Since the parameters satisfy $p>0, u>0, a>u p$, $p^{*}=\lambda p$, and $1>\lambda>0$, the order " $R, T, P>S$ " is supported. Besides, $P=1 / 2 T$ indicates $T>P$. It follows that "The orderR $>S, T>P>$ Sis supported."

(2) Given $R>T, p(a-u p) / 2>p^{*}\left(a-u p^{*}\right)$, denoted as inequality (1), is supported. Since $a>u p$, we can set $a=\beta u p(\beta>1)$. Then, inequality (1) transforms to $(\beta-1) u p^{2}>2 \lambda(\beta-\lambda) u p^{2}$, denoted as inequality (2). Furthermore, since $u p^{2}>0$, inequality (2) transforms to $(\beta-1)>2 \lambda(\beta-\lambda)$, denoted as inequality
(3). It follows that $\beta(1-2 \lambda)>1-2 \lambda^{2}$, denoted as inequality (4), is supported.

While $1>\lambda>0.5$, inequalities $(1-2 \lambda)<0$ and $(1-$ $2 \lambda)<1-2 \lambda^{2}$ are supported. Besides, since $\beta>1$, inequality $\beta(1-2 \lambda)<(1-2 \lambda)$ is supported. Accordingly, $\beta(1-2 \lambda)<1-2 \lambda^{2}$, denoted as inequality (5), is supported. However, inequality (5) contradicts with inequality (4), indicating that "While $1>\lambda>$ $0.5, T>R$ is supported."

While $\lambda=0.5,(\beta-1)<2 \lambda(\beta-\lambda)$ is supported. This contradicts with inequality (3), indicating that "While $\lambda=0.5, T>R$ is supported."

While $0.5>\lambda>0,(1-2 \lambda)>0$ is supported. Inequality (4) transforms to $\beta>\left(1-2 \lambda^{2}\right) /(1-2 \lambda)$. This means that "While0.5 $>\lambda>0, R>$ Tis supported if $\beta$ $>\left(1-2 \lambda^{2}\right) /(1-2 \lambda)$; otherwise, $T>$ Ris supported."

(3) Given $R>P, p(a-u p) / 2>p^{*}\left(a-u p^{*}\right) / 2$, denoted as inequality (6), is supported. With $a=\beta u p$ and $p^{*}=\lambda$ $p$, inequality (6) transforms to $\beta(1-\lambda)>1-\lambda^{2}$. Since $1-\lambda>0, \beta>1+\lambda$ is supported. Thus, "While $\beta>1+$ $\lambda, R>$ Pis supported; otherwise, $P>$ Ris supported."

With the above analysis results, we obtain the order of $R$, $S, T$, and $P$ in Table 1 and present the corresponding evolutionary equilibriums. Full price cutting and full price keeping mean that all firms offer price $p^{*}$ and price $p$, respectively.

It can be seen in Table 1 that under the parameter condition $0.5>\lambda>0$ and $\beta>\left(1-2 \lambda^{2}\right) /(1-2 \lambda)$, the order $R>T>P>S$ is supported and full price keeping emerges as one of the evolutionary equilibriums. Depending on the initial fraction of firms offering price $p$, the price competition system will converge to different evolutionary equilibriums. If the initial fraction $f_{p}(0)$ of firms offering price $p$ is greater than $x^{*}\left(x^{*}=(P-S) /(R-T-S+P)\right)$, that is, $f_{p}(0)>x^{*}$, the system will converge to full price keeping. In reverse, if $f_{p}(0)<x^{*}$, the system will converge to full price cutting. Obviously, there is a critical phenomenon about the price evolutionary equilibrium.

In the study of the evolutionary game on complex networks, a complete network represents the random interactions in a well-mixed situation [32]. Then, the above critical phenomenon should be found by implementing the price competition game on complete networks. For the competitive interactions described by a square lattice, 
TABLE 2: Summary of model parameters for simulation experiment.

\begin{tabular}{lcc}
\hline Parameter & Description & $\begin{array}{c}\text { Values used in } \\
\text { experiments }\end{array}$ \\
\hline$N$ & Competition scale & $1000 \geq N \geq 50$ \\
$p$ & Original price & $1000 \geq p \geq 1$ \\
$p^{*}$ & Cutting price & $p>p^{*}>0$ \\
$u$ & Slope of demand function & $10>u>0$ \\
$a$ & The maximum of demand & $1000 u p \geq a>u p$ \\
\hline
\end{tabular}

small-world network, and scale-free network, the current model is analytically intractable. Thus, in Section 4, we adopt an experimental approach to study its behavior, and analyze the effects of different parameters on price evolution. As a verification of the theory analysis and a necessary comparison to a square lattice, small-world network, and scale-free network, the results on a complete network are also provided.

\section{Simulation Results and Discussion}

All the parameters in our model and their associated ranges for simulation experiments are summarized in Table 2. The justification for such a setting is as follows. First, in the literature studies about social dilemmas on complex networks, such as the prisoner's dilemma game, snowdrift game, and public good game on complex networks, each node in networks represents a person, and the node number is usually set as 5000 or 10,000 . In our model, however, the nodes represent the firms. It is rare in the real world that so many firms engage in price competition for a homogeneous product. Therefore, we set a firm number as 1000 $\geq N \geq 50$, which reflect the appropriate competition scales in the real world. Second, the original price $p$ is greater than 0 , but we cannot examine an infinite $p$ in a simulation experiment. As such, we set it as $1000 \geq p \geq 1$, which is wide enough to explore its effect on price evolution. Similarly, parameters $u$ and $a$ are set as $10>u>0$ and $1000 u p \geq a>u p$, respectively.

In all simulations, the initial prices ( $p$ or $p^{*}$ ) are randomly assigned among all firms with equal probability, and no initial advantage is given to price $p$ or $p^{*}$. Accordingly, the frequency $f_{p}(t)$ of firms keeping original price $p$ among all firms at game time $t$ for the initial state is about 0.5 (i.e., $f_{p}$ $(0) \approx 0.5)$. Then, the price evolution proceeds according to the model described in Section 2, so that the frequency $f_{p}(t)$ changes continually. We monitor the value of $f_{p}(t)$ at each time step. If the value stays unchanged after 50 time steps, the simulation stops and the final value represents the frequency $F_{p}$ of firms keeping the original price in equilibrium state. Otherwise, the simulation runs for 11,000 time steps, and $F_{p}$ is obtained by averaging the frequency $f_{p}(t)$ over the last 1000 time steps, that is, $F_{p}=1 / 1000, \sum_{t=10,001}^{11,000}$ $f_{p}(t)$. In what follows, $F_{p}$ is referred to as "the density of price keeping" for short.
4.1. Emergence of Price Keeping. In Figure 1, we show the probability distribution of the density of price keeping (i.e., $F_{p}$ ) based on 20,000 simulations on a complete network, small-world network, scale-free network, and square lattice, respectively. In each simulation, the accumulated payoff or average payoff is randomly indicated, and the parameters $\left(N, p, p^{*}, u\right.$, and $\left.a\right)$ are generated by randomly sampling them from their associated ranges.

An outstanding feature of the distribution in Figure 1 is that there are four large spikes. The first one is at $F_{p}=0$, which corresponds to the solution of the Bertrand model, that is, the equilibrium state of full price cutting. The probabilities here indicate that the price-keeping phenomena emerge in $37 \%, 59 \%, 87 \%$, and $100 \%$ of all the simulation realizations for the complete network, small-world network, scale-free network, and square lattice, respectively. The second one is at $F_{p}=(0,0.1]$, which means that only no more than $10 \%$ of firms keep the price. The probabilities here indicate that the small-world network, scalefree network, and square lattice are prominent for price keeping in a fierce competition environment. The third one and the fourth one are at $F_{p}=(0.9,1)$ and $F_{p}=1$, respectively. The probabilities here indicate that while the square lattice has little advantage to achieve full price keeping, the small-world network and scale-free network exhibit a stronger capability to facilitate full price keeping than the complete network.

Based on the above results, we know that although the price-keeping phenomena emerge in four different network styles, the small-world network and scale-free network are more beneficial for price keeping than the complete network and square lattice. The complex competitive relation networks in the real world generally have a small-world property and scale-free degree distribution at the same time [41-43]. Therefore, the results in Figure 1 demonstrate the positive effect of complex competitive relation networks on price keeping.

The above results are derived from the combination of all parameters $\left(N, p, p^{*}, u\right.$, and $\left.a\right)$ and two payoff schemes. In the following subsections, we make further exploration on the effects of different parameters and payoff schemes on price evolution. Using Monte Carlo (MC) simulations, we calculate the average level of density $F_{p}$. Each data point of $F_{p}$ in the following results is the average of 100 densities of $F_{p}$ for the same simulation parameter.

4.2. Effects of Payoff Scheme and Competition Scale on Price Evolution. Under the two different payoff schemes, we first explore the level of density $F_{p}$ as a function of competition scale $(N)$, that is, the number of firms in a competitive environment. The results are shown in Figure 2.

It can be observed in Figures 2(a)-2(h) that under two different payoff schemes, density $F_{p}$ is nearly kept unchanged on the complete network, small-world network, and square lattice, but it changes significantly on the scale-free network. More specifically, for the high price cutting (i.e., (a), (b), (e), and (f)), the density $F_{p}$ on the scale-free network under the 


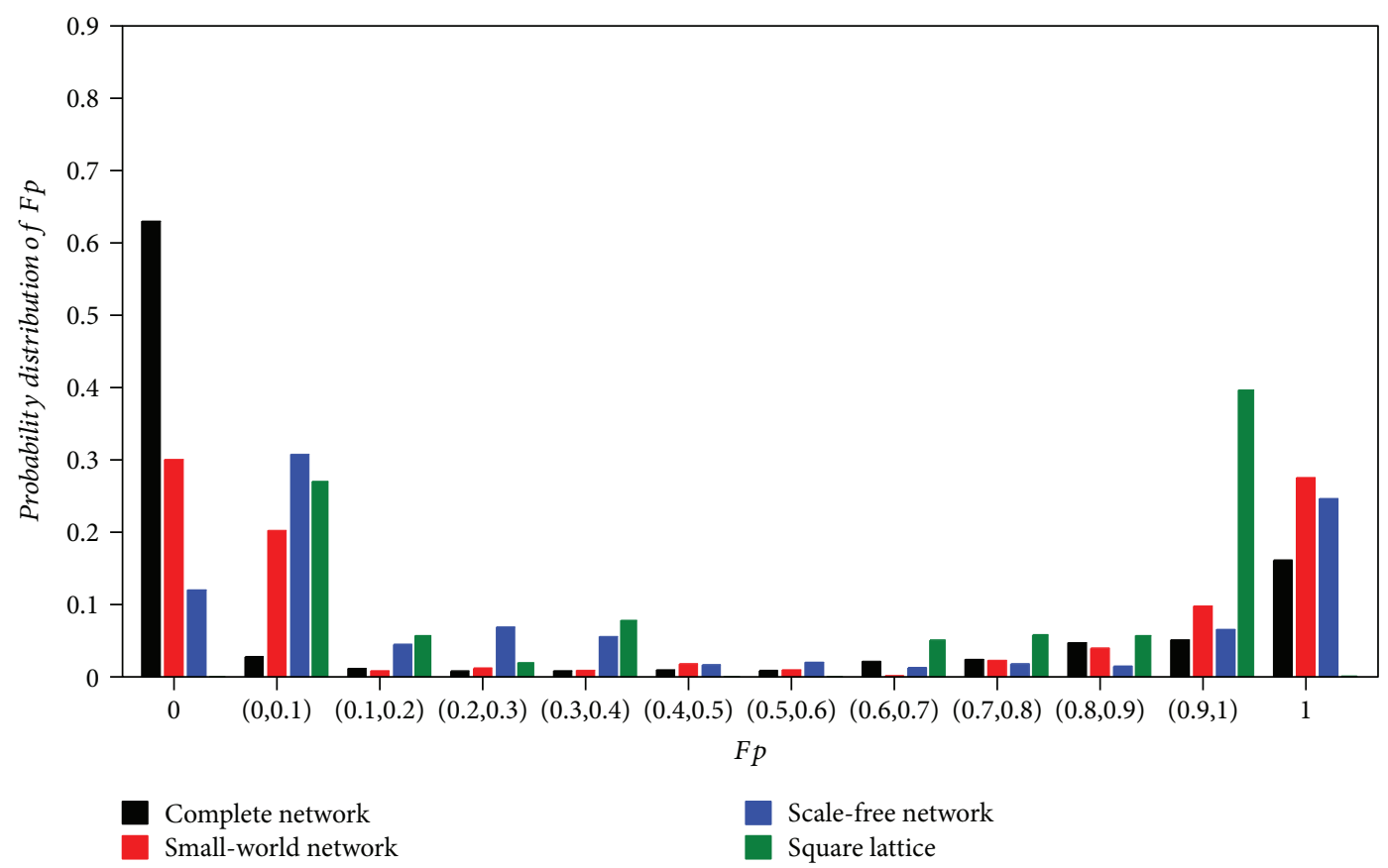

FIGURE 1: Probability distribution of the density of price-keeping $F_{p}$ on four different network styles.

average payoff is higher than that under the accumulated payoff. Nevertheless, for the low price cutting (i.e., (c), (d), (g), and (h)), the situation is reversed. Such a result confirms the previous finding that the evolutionary dynamics on the scale-free network is affected by payoff schemes [31, 4648]. The main reason is that the game payoffs of hub nodes in the scale-free network are noticeably different under different payoff schemes. Figure 3 shows the payoffs of firms with different degrees on scale-free networks during the dynamic evolutionary process under two different payoff schemes. The data with different marks in Figures 3(a) and 3(b) come from 10 different simulation experiments. It is clear that, under an accumulated payoff scheme, the game payoffs of hub nodes are much higher than those of low degree nodes, but under an average payoff scheme, hub nodes have little advantage in obtaining high game payoffs. According to the price evolutionary mechanism described in Section 2, game payoff is a crucial factor in the imitation behaviors of firms. Therefore, such a change in game payoff of hub nodes inevitably results in different imitation dynamics and evolutionary results.

Moreover, in Figures 2(a)-2(h), a common tendency is that density $F_{p}$ increases with competition scale $N$ except for extremely low values $\left(F_{p} \rightarrow 0\right)$ or high values $\left(F_{p} \rightarrow 1\right)$. Such a result is consistent with the finding obtained in the literature where individuals play PDG on adaptive networks [59], and it is indicated that a big competition scale is beneficial for price keeping in a competition environment.

Based on the above results, we attempt to specify the effects of the other four parameters $\left(p, p^{*}, u\right.$, and $\left.a\right)$ on price evolution under three levels of competition scales, that is, low, middle, and high scales. For the scale-free network, we provide the results under two different payoff schemes. For complete networks, small-world networks, and square lattices, we present the results under an accumulated payoff, but verify the results under an average payoff.

4.3. Effects of Price Cutting $p^{*}$ and Demand Quantity a on Price Evolution. Figure 4 shows the effects of price cutting $p^{*}$ and demand quantity $a$ on price evolution along the $p^{*}$-axis and $a$-axis, respectively.

It can be seen in Figure 4 that under different competition scales and payoff schemes, density $F_{p}$ decreases with $p^{*}$ on four network styles, and a high level of $F_{p}$ is achieved when $p^{*}$ is low. This means that it is easier for the price competition system to maintain the original price while the price cutting behavior of some firms is more severe at the beginning. However, the effect of demand quantity $a$ on density $F_{p}$ is not significant. Besides, a critical phenomenon predicted by theoretical analysis in Section 3 appears at $p^{*}=3,4$ in complete networks with $N=500$ and $N=1000$ except for the demand quantity $a=10$. We understand the above experiment results based on the following reasoning.

Firstly, according to the theoretical analysis in Section 3, the price evolutionary equilibrium depends on the initial fraction $f_{p}(0)$ and the value of $x^{*}$. In our simulation experiment, the initial fraction $f_{p}(0) \approx 0.5$. Therefore, the value of $x^{*}$ is crucial for the evolutionary results. With $R=p(a-u p) /$ 2, $T=p^{*}\left(a-u p^{*}\right), P=p^{*}\left(a-u \cdot p^{*}\right) / 2, S=0, a=\beta u p$, and $p^{*}=\lambda p$, the equation $x^{*}=(P-S) /(R-T-S+P)=P /(R-$ $P)=\left(\lambda(\beta-\lambda) u p^{2}\right) /\left((\beta-1) u p^{2}-\lambda(\beta-\lambda) u p^{2}\right)$ is supported. In Figure 4, the effects of $p^{*}$ and $a$ on the density of pricekeeping $F_{p}$ are investigated by fixing $p$ and $u$ at $p=10$ and $u=0.1$. Then, the value of $x^{*}$ is just decided by $\beta(\beta>1)$ and $\lambda(1>\lambda>0)$, that is, $x^{*}=\lambda(\beta-\lambda) /((\beta-1)-\lambda(\beta-\lambda))$. We show the relation between $x^{*}$ and $\beta, \lambda$ in Figure 5 . 


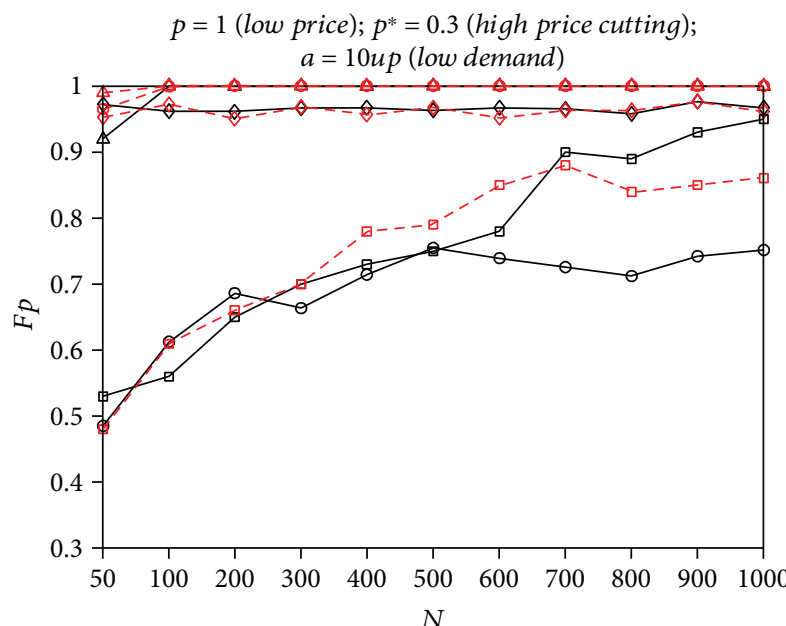

$\rightarrow$ Complete network/accumulated payoff

$\triangle$ Small-world network/accumulated payoff

- Scale-free network/accumulated payoff

$\rightarrow$ Lattice/accumulated payoff

- - - Complete network/average payoff

$-\Delta-$ Small-world network/average payoff

$-\theta$ - Scale-free network/average payoff

$-\diamond-$ Square lattice/average payoff

(a)

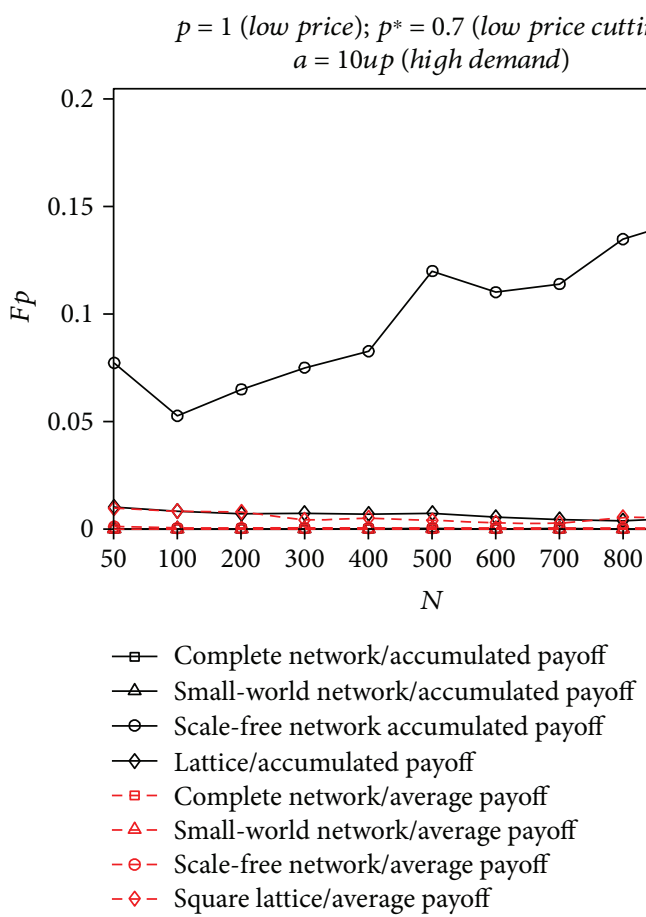

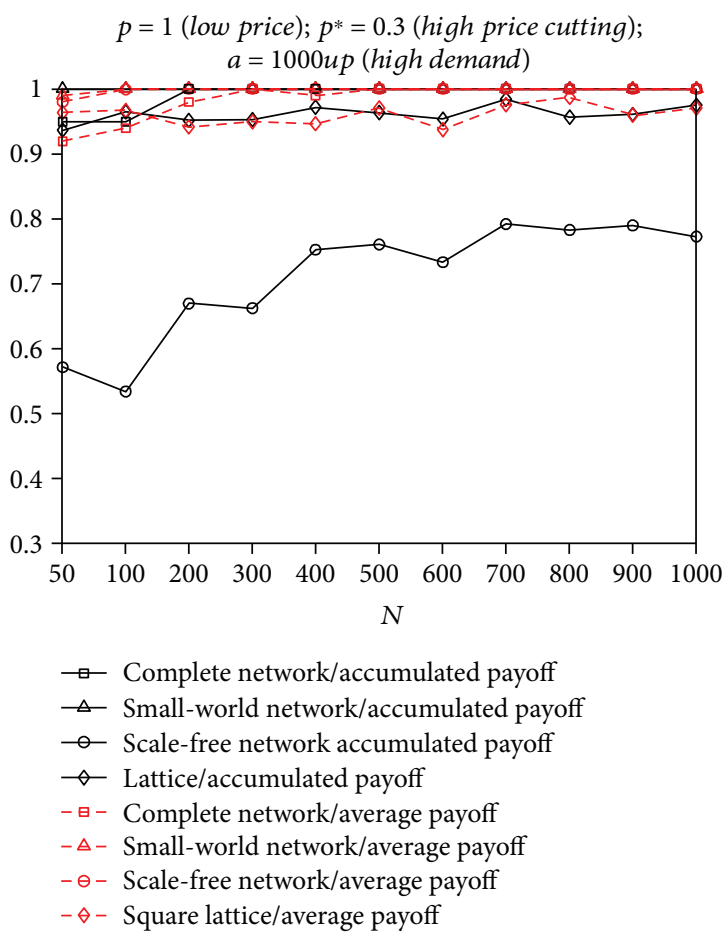

(b)

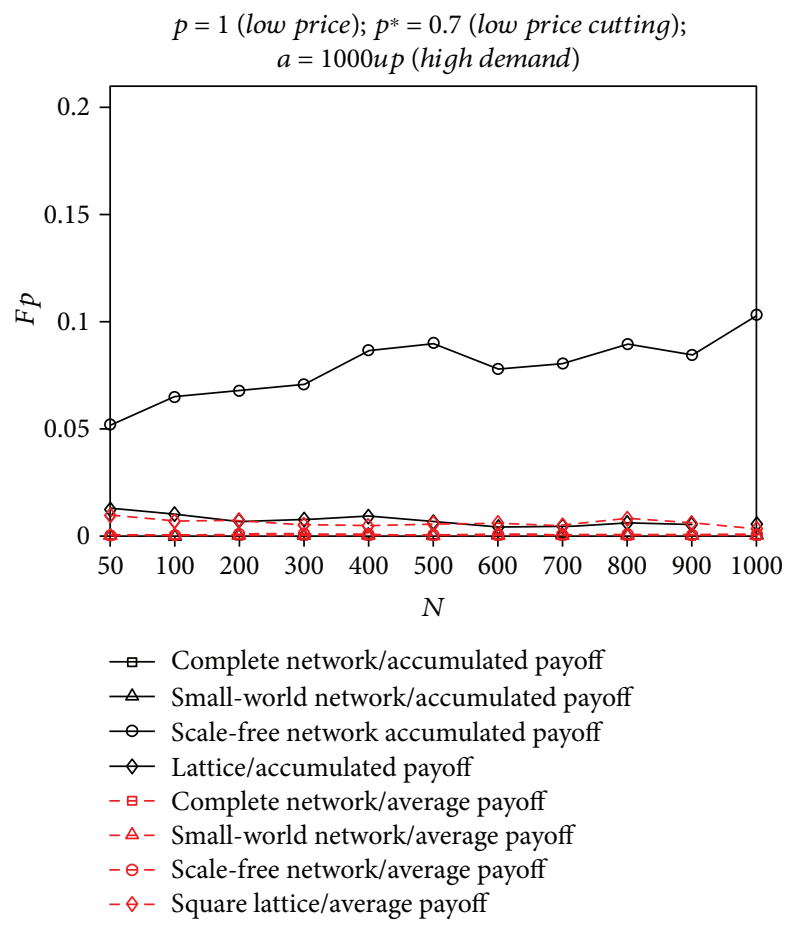

FIgURe 2: Continued. 


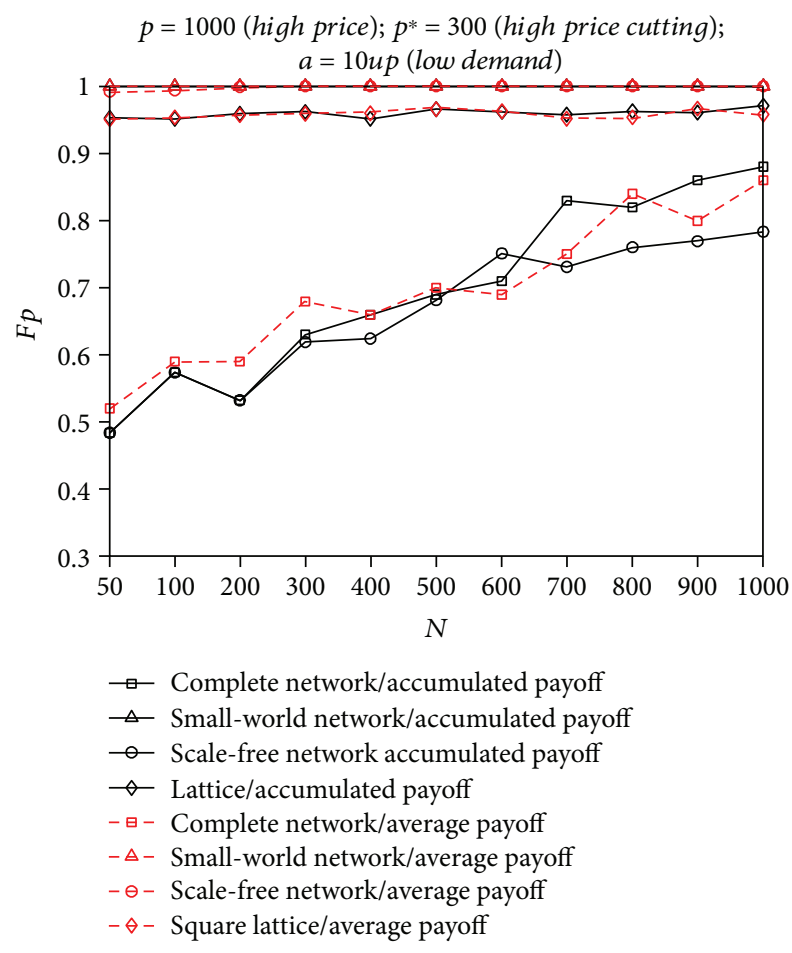

(e)

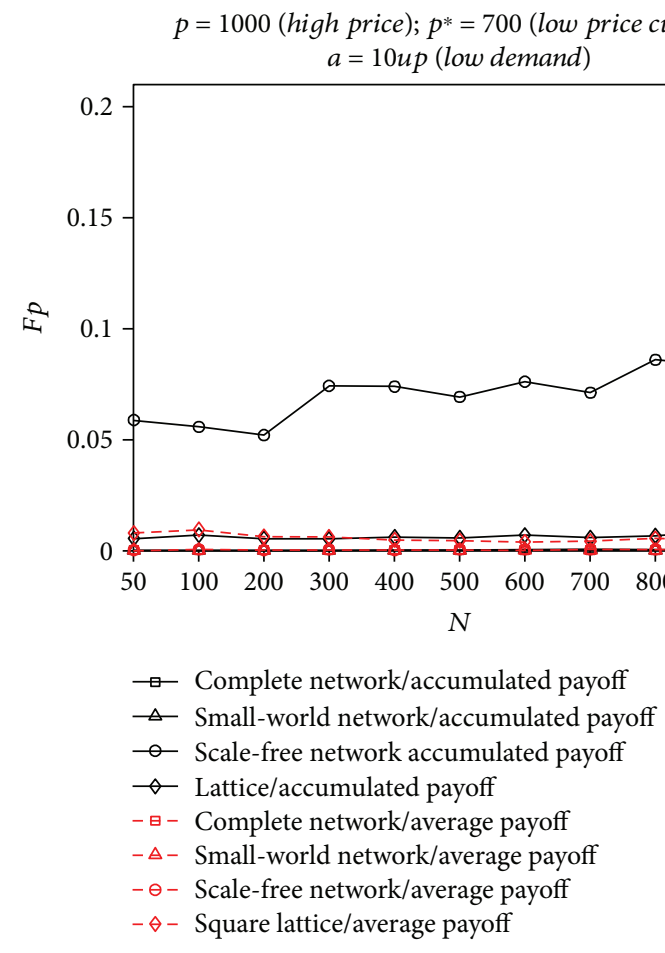

(g)

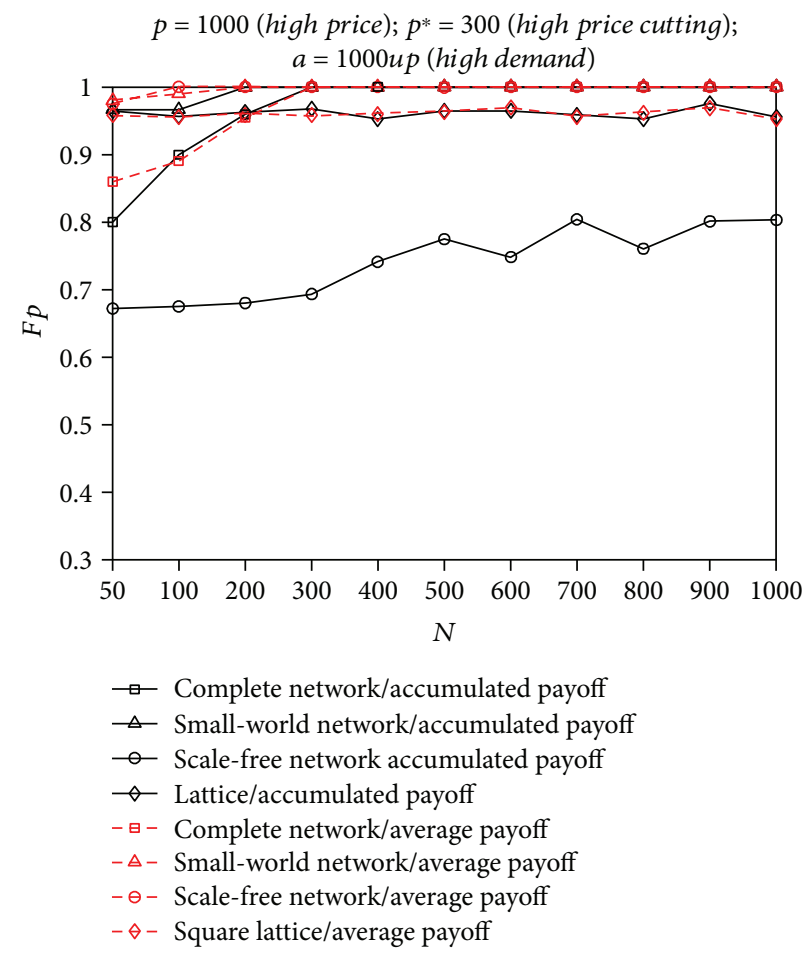

(f)

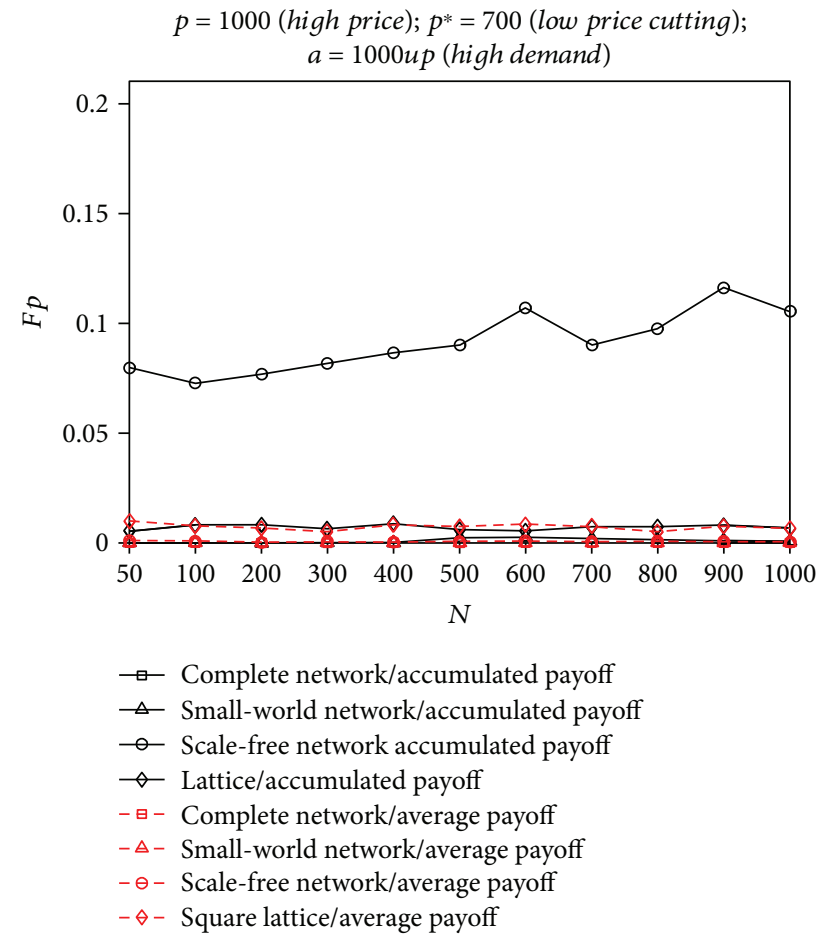

(h)

Figure 2: Density of price keeping, $F_{p}$, as a function of competition scale $N$ for specific parameter combinations (a) $p=1, p^{*}=0.3$, and $a=10 u p$, (b) $p=1, p^{*}=0.3$, and $a=1000 u p$, (c) $p=1, p^{*}=0.7$, and $a=10 u p$, (d) $p=1, p^{*}=0.7$, and $a=1000 u p$, (e) $p=1000, p^{*}=300$, and $a=10 u p$, (f) $p=1000, p^{*}=300$, and $a=1000 u p$, (g) $p=1000, p^{*}=700$, and $a=10 u p$, and (h) $p=1000, p^{*}=700$, and $a=1000 u p$. For all the cases, the slope of the demand function is set as $u=1$. Note that for the competition scales $N=50 \sim 1000$, the number of firms on the square lattice are $N=49,100,186,289,400,484,576,676,784,900$, and 1024 , and the corresponding dimensions of the square lattices are $7,14,17,20,22,24,26,28,30$, and 32 , respectively. 


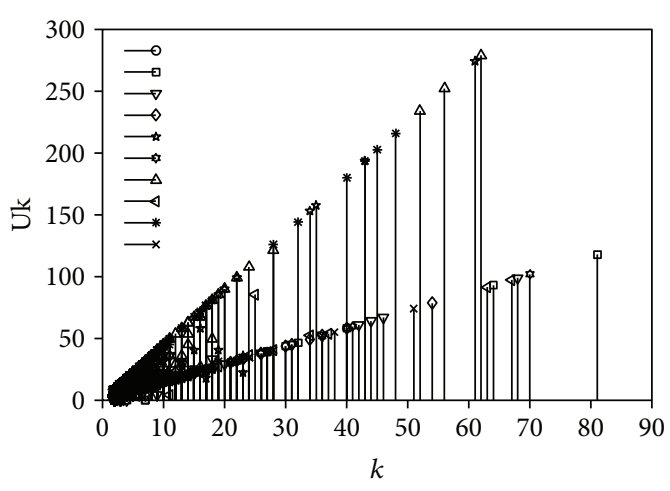

(a)

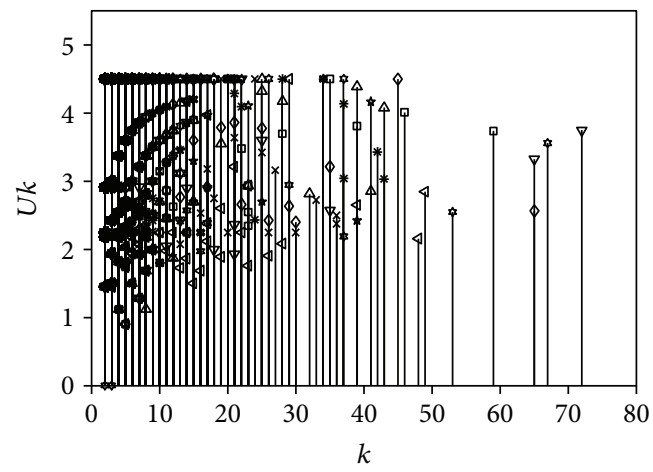

(b)

Figure 3: The payoffs of firms with a different degree $k$ during the dynamic evolutionary process under (a) accumulated payoff and (b) average payoff. Note that in both cases, parameters $p=1, p^{*}=0.3, a=10 u p$, and $u=1$, corresponding to the parameter setting in Figure 2(a). Population is $N=500$.

It can be seen in Figure 5 that $x^{*}$ increases with $\lambda$, but hardly changes with $\beta$. As such, while $p^{*}$ increases, the value of $\lambda$ increases, and $x^{*}$ increases accordingly. This means that for a given $f_{p}(0) \approx 0.5$, the price keeping is more difficult to achieve under a higher $p^{*}$. Thus, it can be seen from Figure 4 that the density of price-keeping $F_{p}$ decreases with $p^{*}$. On the other hand, with the increase of $a$, the value of $\beta$ increases, but the value of $x^{*}$ is not affected. As a result, although the value of $a$ increases significantly, the density of price-keeping $F_{p}$ nearly stays the same.

Secondly, in Table 3, we list the values of $x^{*}$ for $p^{*}=3,4$ (i.e., $\lambda=0.3,0.4$ ), where a critical phenomenon of $F_{p}$ is observed in complete networks with $N=500$ and $N=1000$ in Figure 4. All data are accurate to three digits after the decimal point.

It can be seen in Table 3 that while $p^{*}$ changes from $p^{*}=3$ to $p^{*}=4$, the values of $x^{*}$ change from $x^{*}<0.5$ to $x^{*}>0.6$. Based on the analysis in Section 3, it can be obtained that if the initial fraction $f_{p}(0) \approx 0.5$ is less than $x^{*}$, full price cutting is the evolutionary equilibrium. Then, $p^{*} \geq 4$ corresponds to $x^{*}>0.6$, and thus $f_{p}(0)<x^{*}$ is inevitable in 100 repeated simulation experiments. As a result, full price cutting is achieved on complete networks for $p^{*} \geq 4$. Inversely, while $p^{*}=3$, the value of $f_{p}(0) \approx 0.5$ is larger than $x^{*}$, full price keeping is the evolutionary equilibrium according to the theoretical analysis. However, for the demand quantity $a=10$, the value of $f_{p}(0) \approx 0.5$ is close to $x^{*}$. Then, it is possible that $f_{p}(0)<x^{*}$ happens several times in 100 repeated simulation experiments. As a result, in Figures 4(b) and $4(c)$, we observe a high level of density of price keeping, but not full price keeping for $p^{*}=3$ and $a=10$. In Figure 4(a), the anticipated full price keeping is not achieved for $p^{*}=3$. The discrepancy can be attributed to the difference in competition scale. The theoretical analysis in Section 3 is suitable for a large competition scale, which is difficult to be satisfied for $N=50$ in Figure 4(a).

4.4. Effects of Original Price $p$ and Demand Slope $u$ on Price Evolution. According to the results in Section 4.3, demand quantity $a$ has little effect on price evolution. Therefore, in this experiment it is fixed as $a=1000$, which is large enough that $D\left(p_{i}\right)=a-u \cdot p_{i}>0$ can be satisfied while the values of parameters $p$ and $u$ change. Then, we choose Figures 4(a), $4(\mathrm{e}), 4(\mathrm{i}), 4(\mathrm{k})$, and 4 (o) to make a further experiment exploration, which covers three competition scales, four network styles, and two payoff schemes. For each subgraph, we set $p^{*}=3,4,5$, which covers the main characteristics of $F_{p}$ shown in Figure 4.

Figure 6 shows the effects of original price $p$ and demand slope $u$ on price evolution along $p$-axis and $u$-axis, respectively.

It can be seen in Figure 6 that under different competition scales and payoff schemes, both original price and demand slope have significant influence on the density $F_{p}$ on four network styles. For the low demand slopes (e.g., $u<25$ ), $F_{p}$ increases with $p$, and a high level of $F_{p}$ is achieved when $p$ is high. Nevertheless, for some higher slope values of $u$, $F_{p}$ increases with $p$ at the beginning and then decreases with it. If the slope $u$ is extremely high, it is difficult for the whole system to keep the price. Besides, $F_{p}$ decreases with demand slope $u$ for various original prices $p$. We understand these results in the following.

Firstly, with $R=p(a-u p) / 2, \quad T=p^{*}\left(a-u p^{*}\right), \quad P=p^{*}$ $\left(a-u \cdot p^{*}\right) / 2, S=0$, the equation $x^{*}=p^{*}\left(a-u p^{*}\right) / p(a-u$ $p)-p^{*}\left(a-u p^{*}\right)$ is supported. While parameters $p^{*}, a$, and $u$ keep invariable, the numerator $p^{*}\left(a-u p^{*}\right)$ is constant and greater than 0 . Then, the value of $x^{*}$ is just decided by the denominator $p(a-u p)-p^{*}\left(a-u p^{*}\right)$. For the function $y=p(a-u p)-p^{*}\left(a-u p^{*}\right)$, differential coefficients $d y / d p=$ $a-2 u p$ and $d^{2} y / d p^{2}=-2 u$ are supported. Since $u>0, d^{2}$ $y / d p^{2}<0$ is supported. Then, the function $y=p(a-u p)-$ $p^{*}\left(a-u p^{*}\right)$ is convex with the parameter $p$, and its maximum is achieved while $p=a / 2 u$. Accordingly, $x^{*}=$ $p^{*}\left(a-u p^{*}\right) /\left(p(a-u p)-p^{*}\left(a-u p^{*}\right)\right)$ is concave with $p$ and its minimum is achieved while $p=a / 2 u$. Then, for a given initial fraction $f_{p}(0) \approx 0.5$, the density of price-keeping $F_{p}$ is convex with $p$, that is, $F_{p}$ increases with $p$ initially, peaks at $p=a / 2 u$, and then decreases with it. Such an analysis result is verified by the experiment data of $F_{p}$ for some slope values 


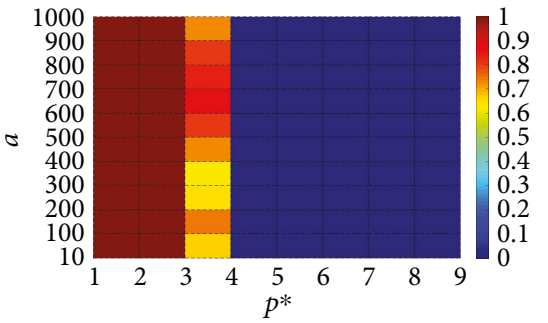

(a)

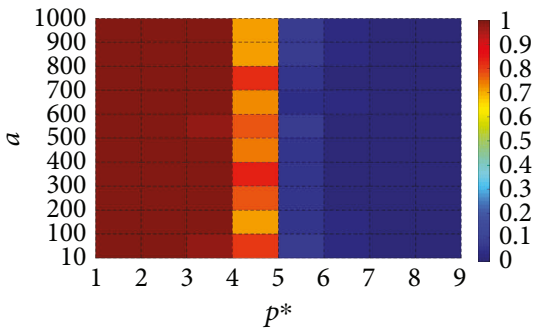

(d)

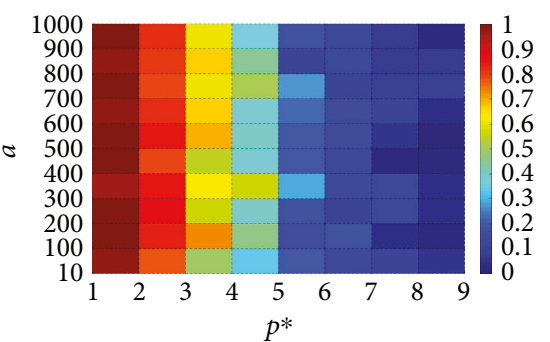

(g)

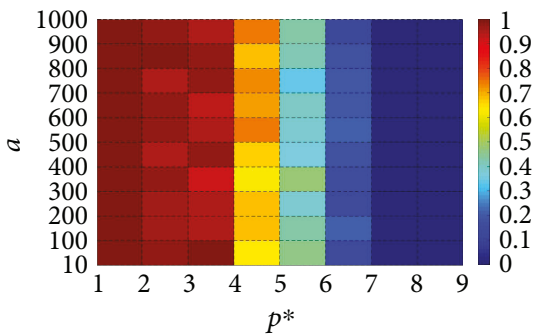

(j)

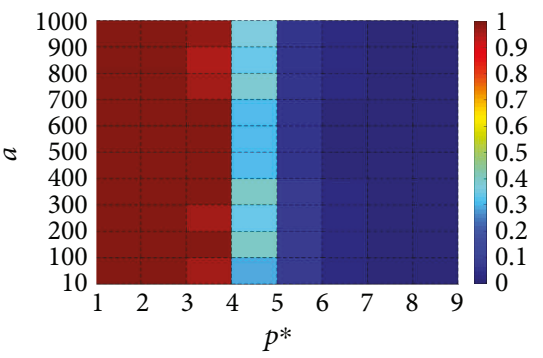

(m)

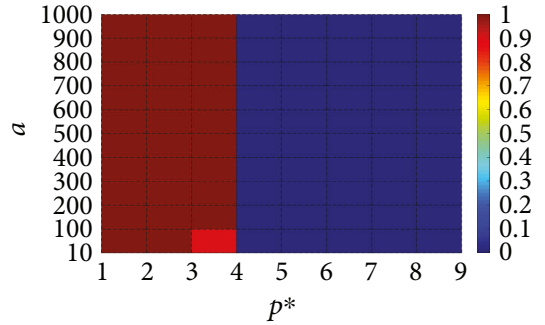

(b)

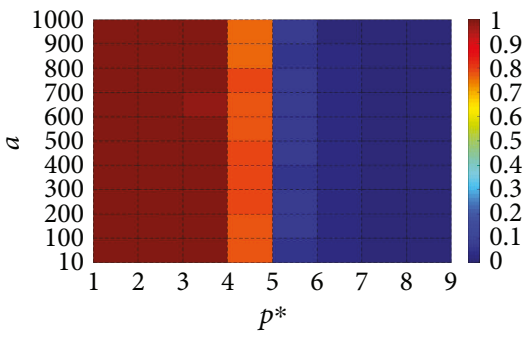

(e)

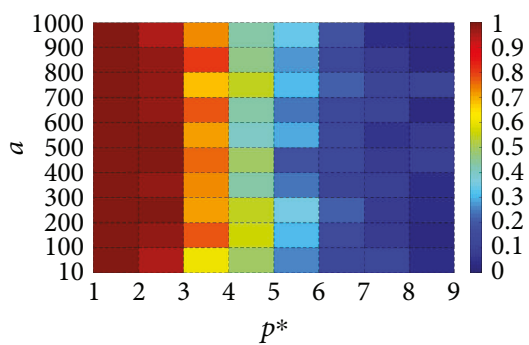

(h)

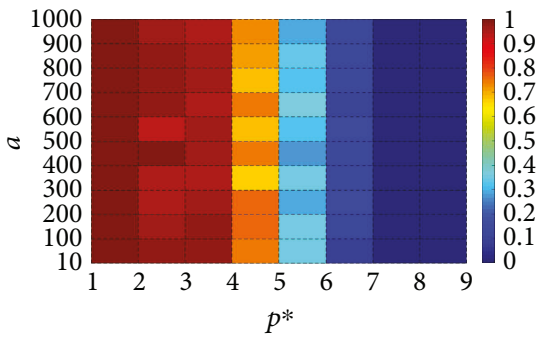

(k)

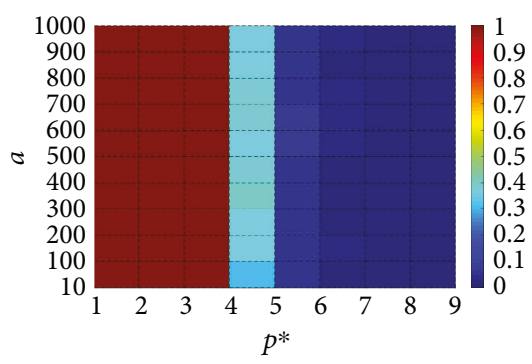

(n)

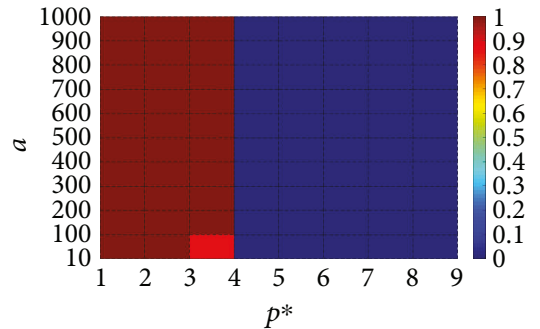

(c)

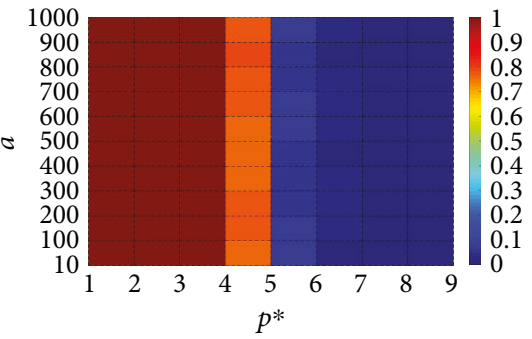

(f)

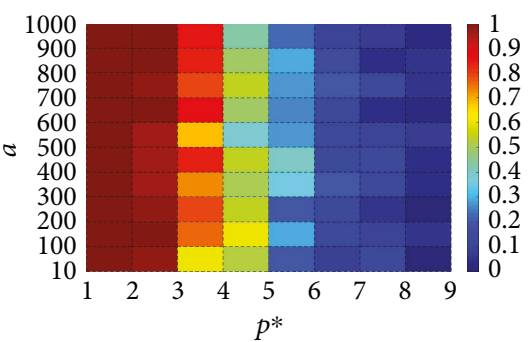

(i)

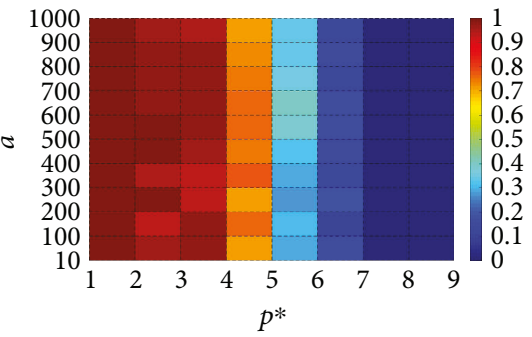

(1)

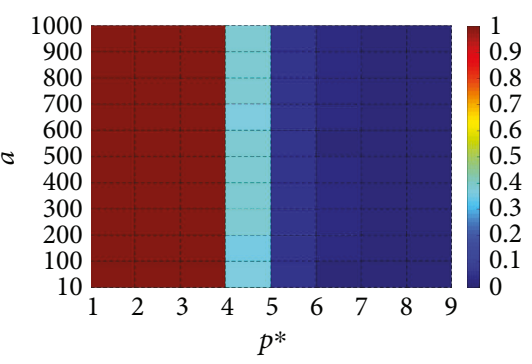

(o)

FIgURE 4: Density of price keeping, $F_{p}$, as a function of price cutting $p^{*}$ and demand quantity $a$ along the $p^{*}$-axis and $a$-axis, respectively, in (a) a complete network with $N=50$, (b) complete network with $N=500$, (c) complete network with $N=1000$, (d) small-world network with $N=50$, (e) small-world network with $N=500$, (f) small-world network with $N=1000$, (g) scale-free network with $N=50$, (h) scale-free network with $N=500$, (i) scale-free network with $N=1000$, (j) square lattice with $N=49(7 \times 7)$, (k) square lattice with $N=484(22 \times 22)$, (l) square lattice with $N=1024(32 \times 32)$, (m) scale-free network with $N=50$ under an average payoff, (n) scale-free network with $N=500$ under an average payoff, and (o) scale-free network with $N=1000$ under an average payoff. From (a) to (l), an accumulated payoff is applied and the results have been verified under an average payoff. Note that $p=10$ and $u=0.1$ in all the cases. 


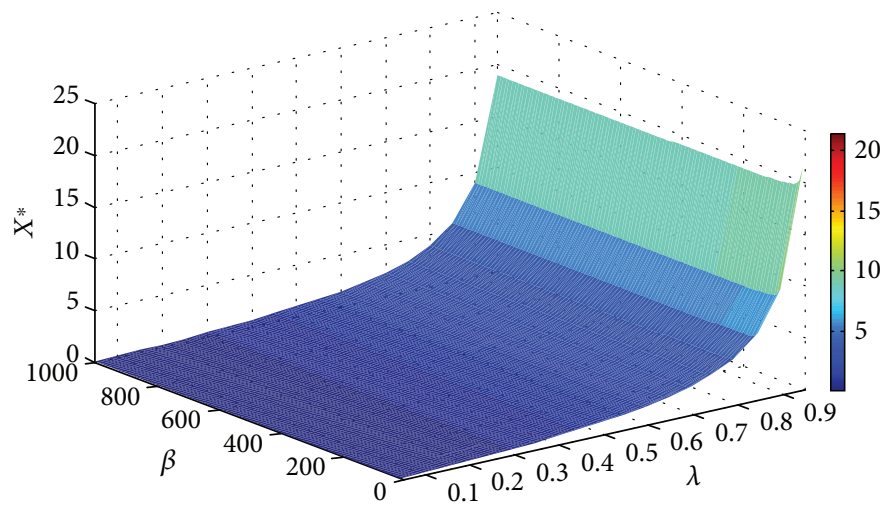

Figure 5: The relation between the values of $x^{*}$ with parameters $\lambda$ and $\beta$ along the $\lambda$-axis and $\beta$-axis, respectively.

TABle 3: Values of $x^{*}$ for price cutting $p^{*}=3,4$.

\begin{tabular}{lccccccccrrr}
\hline $\begin{array}{l}a \\
p^{*}\end{array}$ & 10 & 100 & 200 & 300 & 400 & 500 & 600 & 700 & 800 & 900 & 1000 \\
\hline 3 & $\mathbf{0 . 4 7 8}$ & $\mathbf{0 . 4 3 3}$ & $\mathbf{0 . 4 3 1}$ & $\mathbf{0 . 4 3 0}$ & $\mathbf{0 . 4 3 0}$ & $\mathbf{0 . 4 2 9}$ & $\mathbf{0 . 4 2 9}$ & $\mathbf{0 . 4 2 9}$ & $\mathbf{0 . 4 2 9}$ & $\mathbf{0 . 4 2 9}$ & $\mathbf{0 . 4 2 9}$ \\
4 & $\mathbf{0 . 7 4 4}$ & $\mathbf{0 . 6 7 3}$ & $\mathbf{0 . 6 7 0}$ & $\mathbf{0 . 6 6 9}$ & $\mathbf{0 . 6 6 9}$ & $\mathbf{0 . 6 6 9}$ & $\mathbf{0 . 6 6 8}$ & $\mathbf{0 . 6 6 8}$ & $\mathbf{0 . 6 6 8}$ & $\mathbf{0 . 6 6 7}$ & $\mathbf{0 . 6 6 7}$ \\
\hline
\end{tabular}

of $u \geq 25$ in Figure 6. For the slope values less than 25, we cannot observe such a theoretical analysis result. This can be reasonably explained as follows. While the value of $u$ is low, the value of $p=a / 2 u$ is high. Accordingly, $F_{p}$ increases with $p$ under a broad range of $p$. Thus, for the low values of $u$ in Figure 6, the only observation is the increase of $F_{p}$ with $p$. In order to verify such an explanation, we make further simulation experiments for the low values of $u<25$. With the continuously increased value of $p$, we indeed find the decrease of $F_{p}$ with $p$.

Secondly, we transfer the equation $x^{*}=p^{*}\left(a-u p^{*}\right) /$ $\left(p(a-u p)-p^{*}\left(a-u p^{*}\right)\right)$ to $x^{*}=\left(a / p^{*}-u\right) /\left(a\left(p-p^{*}\right) / p^{* 2}\right.$ $\left.-u\left(\left(p^{2}-p^{* 2}\right) / p^{* 2}\right)\right)$. While parameters $p^{*}, a$, and $p$ keep invariable, $a / p^{*}, a\left(p-p^{*}\right) / p^{* 2}$, and $\left(p^{2}-p^{* 2}\right) / p^{* 2}$ are all constant.Given $A=a / p^{*}, B=a\left(p-p^{*}\right) / p^{* 2}$, and $C=\left(p^{2}-p^{* 2}\right) / p^{* 2}$, we transfer $x^{*}=a / p^{*}-u /\left(a\left(p-p^{*}\right) / p^{* 2}-u\left(\left(p^{2}-p^{* 2}\right) / p^{* 2}\right)\right)$ to $x^{*}=(A-u) /(B-C u)=1 / C(1+(A C-B) /(B-C u))$. Since $1 / C$ is constant, the value of $x^{*}$ is decided by $(A C-B) /$ $(B-C u)$. Furthermore, the numerator $A C-B=\left(a p^{2}-\right.$ $\left.a p p^{*}\right) / p^{* 3}$ is constant and greater than 0 , and then the value of $x^{*}$ increases with the demand slope $u$. As a result, for a given initial fraction $f_{p}(0) \approx 0.5$, the density of $F_{p}$ decreases with demand slope $u$, as observed in Figure 6. This means that if the demand of a product is sensitive to price change, it is difficult for all the firms to keep the original price.

\section{Conclusions}

The evolutionary game is the theory of dynamic adaption and learning in repeated games played by bounded rational players. It is one of the key paradigms behind many scientific disciplines from biology to behavioral sciences to economics. When the interacting players in a game are linked in a specific complex network style, the evolutionary game on complex networks, which integrates the evolutionary game theory and complex network theory, provides an effective method to obtain the solution of the game.

In this work, based on the Bertrand model, we study the price competition problem from the perspective of the evolutionary game on complex networks. To the best of our knowledge, this is the first work that attempts to understand the price competition problem under the consideration of the complex competition relations among the firms who charge the price. We find that once the firms are considered as a bounded rationality and just make a simple decision on keeping the price or cutting the price, full price keeping is one of the evolutionary equilibriums. More importantly, the pricekeeping phenomenon emerges in the small-world network, scale-free network, and square lattice much more frequently than in the complete network. In the fierce competition environment where more than $90 \%$ of the firms cut their price, the small-world network, scale-free network, and square lattice are a prominently beneficial example for the other less than $10 \%$ of the firms to keep the price. While the square lattice has little advantage to achieve full price keeping, the small-world network and scale-free network exhibit a stronger capacity in full price keeping than the complete network. These results indicate that the complex competition relation among firms is a crucial factor to maintain the price in the real world. Besides, competition scale, original price, degree of price cutting, and demand sensitivity to price also influence the price evolution. Specifically, the larger the competition scale, the easier for the whole system to keep the original price; the more severe the price cutting of some firms at the beginning, the easier for all the firms to keep the original price at the evolutionary equilibrium state; the more sensitive the demand to the price cutting, the more difficult for all the firms to keep the original price. The effect of original price on price evolution is relatively complex. Both extremely low and high original prices are not beneficial for price keeping. There exists a medium range of original price under which an 


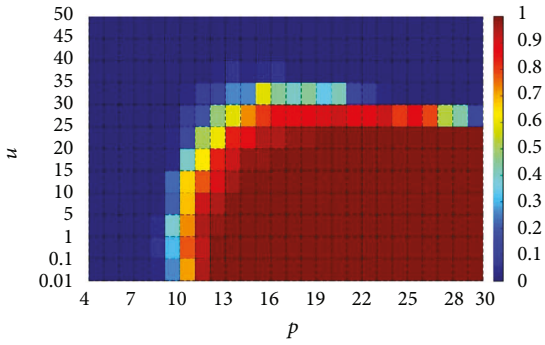

(a)

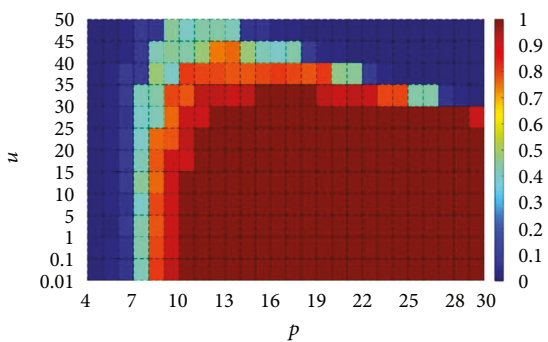

(d)

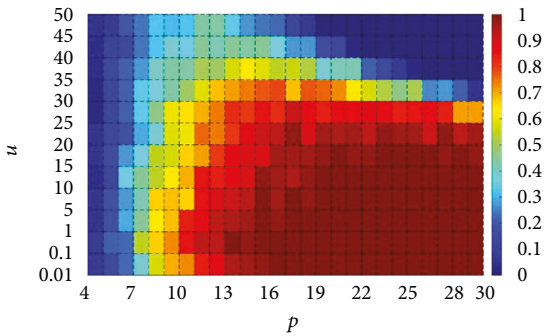

(g)

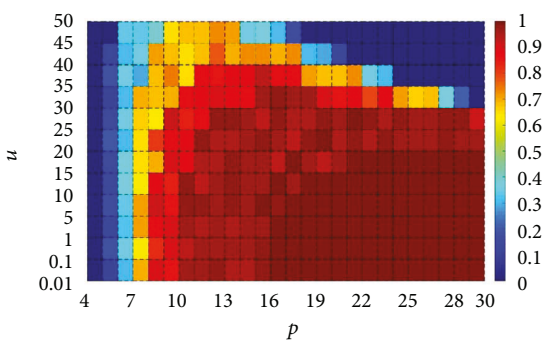

(j)

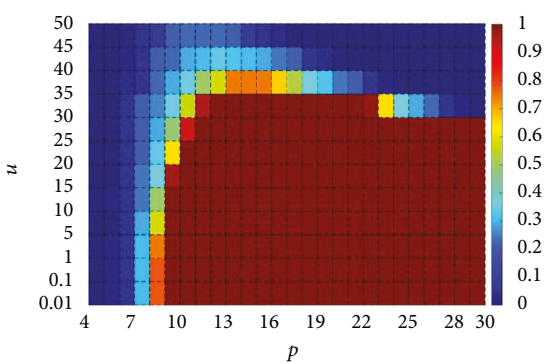

(m)

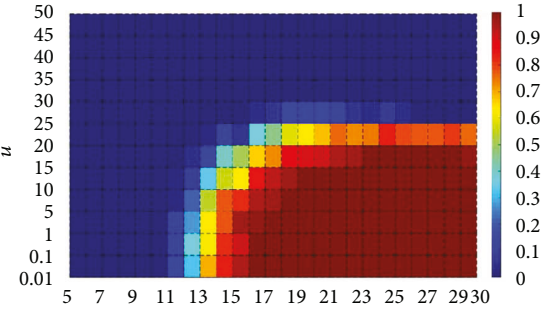

(b)

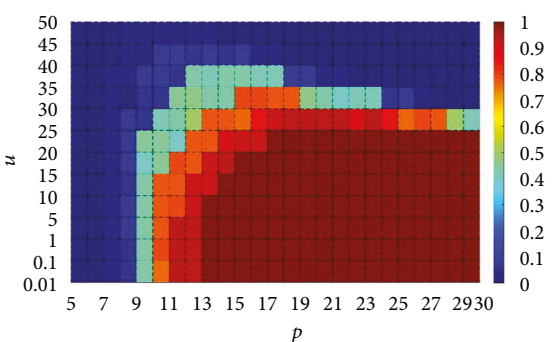

(e)

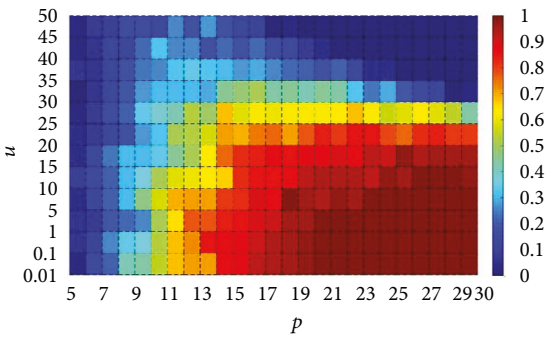

(h)

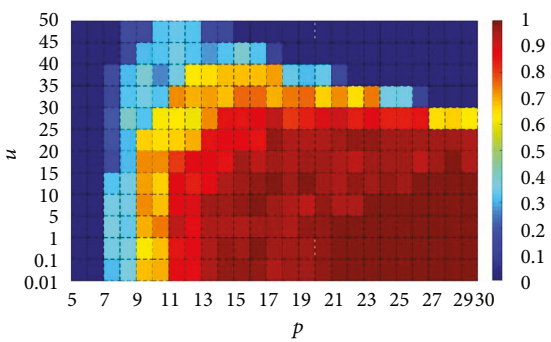

(k)

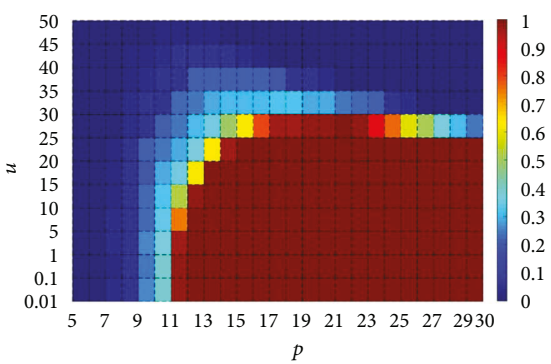

(n)

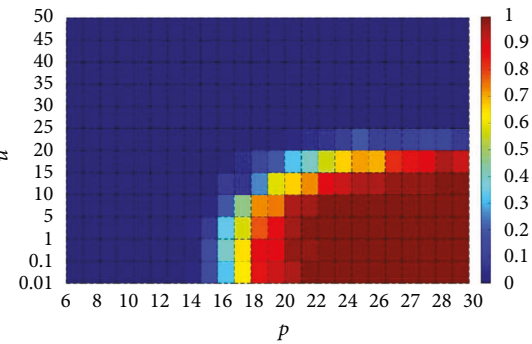

(c)

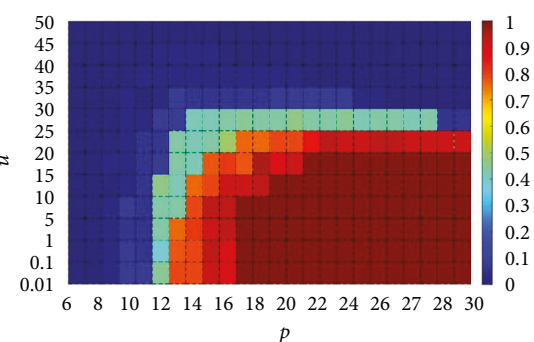

(f)

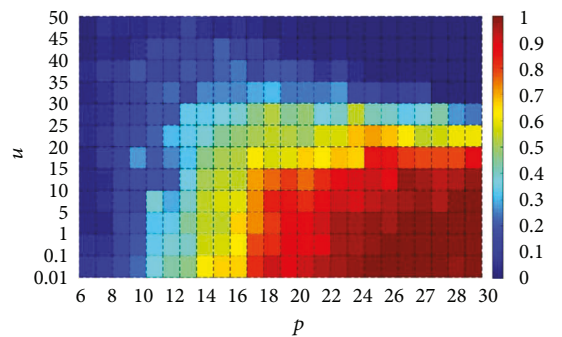

(i)

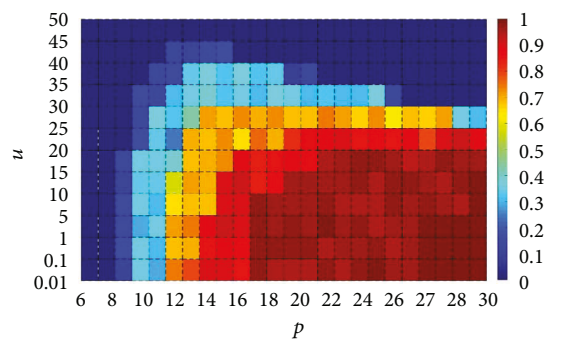

(l)

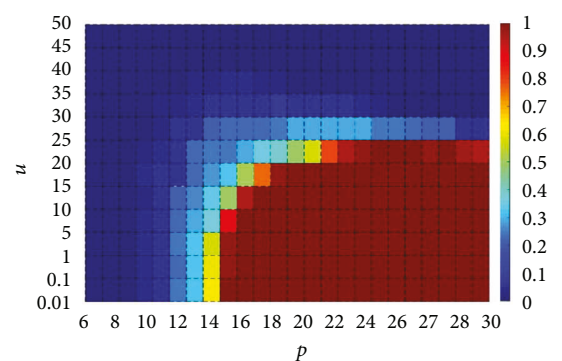

(o)

FIGURE 6: Density of price-keeping, $F_{p}$, as a function of original price $p$ and demand slope $u$ along the $p$-axis and $u$-axis, respectively, in (a) a complete network with $N=50, p^{*}=3$, (b) complete network with $N=50, p^{*}=4$, (c) complete network with $N=50, p^{*}=5$, (d) small-world network with $N=500, p^{*}=3$, (e) small-world network with $N=500, p^{*}=4$, (f) small-world network with $N=500, p^{*}=5$, (g) scale-free network with $N=1000, p^{*}=3$, (h) scale-free network with $N=1000, p^{*}=4$, (i) scale-free network with $N=1000, p^{*}=5$, (j) square lattice with $N=484(22 \times 22), p^{*}=3$, (k) square lattice with $N=484(22 \times 22), p^{*}=4$, (l) square lattice with $N=484(22 \times 22), p^{*}=5$, (m) scalefree network with $N=1000, p^{*}=3$ under an average payoff, (n) scale-free network with $N=1000, p^{*}=4$ under an average payoff, and (o) scale-free network with $N=1000, p^{*}=5$ under average an payoff. From (a) to (l), the accumulated payoff is applied and the results have been verified under the average payoff. Note that $a=1000$ in all cases. 
optimal price-keeping equilibrium is achieved. Lastly, the payoff scheme, which describes how each firm's payoff is calculated in each round game, influences the price evolution on the scale-free network. Under the accumulated payoff and average payoff, there are significant differences in the density of firms keeping the price in the evolution system. These results provide new and important insights for understanding price competition in the real world.

Based on the current results, we can also envision some important extension work in the future. Firstly, the WS small-world network model and BA scale-free network model are two of the most classic network models in the complex network theory. Several network models are based on the two models for characterizing various structural properties of a complex network, such as degree correlation and community structure and mixing pattern [60]. These structural properties have significant effects on cooperation evolution $[53,54,61,62]$. Then, it would be interesting to investigate the possible influence of running the current price competition model on other network models. Besides, this study adopts a simple linear demand function to describe the relation between demand quantity and price change. It is valuable to explore whether the current results are supported under more complex demand function patterns. These considerations will further advance the study of the price competition problem on complex networks.

\section{Conflicts of Interest}

The authors declare that there is no conflict of interest regarding the publication of this paper.

\section{Acknowledgments}

The authors thank the financial support of the National Social Science Foundation of China (no. 15BGL014) and Social Science Youth Foundation of the Ministry of Education of China (no. 12YJCZH226) and for the comments and suggestions from anonymous reviewers.

\section{References}

[1] A. F. Daughety, Ed., Cournot Oligopoly: Characterization and Applications, Cambridge University Press, 2005.

[2] B. Hehenkamp, "Sluggish consumers: an evolutionary solution to the Bertrand paradox," Games and Economic Behavior, vol. 40, no. 1, pp. 44-76, 2002.

[3] E. Maskin, "The existence of equilibrium with price-setting firms," The American Economic Review, vol. 76, no. 2, pp. 382-386, 1986.

[4] M. Yano, "Coexistence of large firms and less efficient small firms under price competition with free entry," International Journal of Economic Theory, vol. 1, no. 3, pp. 167-188, 2005.

[5] M. Yano and T. Komatsubara, "Endogenous price leadership and technological differences," International Journal of Economic Theory, vol. 2, no. 3-4, pp. 365-383, 2006.

[6] H. Hotelling, "Stability in competition," The Economic Journal, vol. 39, no. 153, pp. 41-57, 1929.
[7] N. Singh and X. Vives, "Price and quantity competition in a differentiated duopoly," The Rand Journal of Economics, vol. 15, no. 4, pp. 546-554, 1984.

[8] M. Janssen and E. Rasmusen, "Bertrand competition under uncertainty," The Journal of Industrial Economics, vol. 50, no. 1, pp. 11-21, 2002.

[9] M. Reisinger and L. Ressner, "The choice of prices versus quantities under uncertainty," Journal of Economics \& Management Strategy, vol. 18, no. 4, pp. 1155-1177, 2009.

[10] T. G. Smith and A. Tasnádi, "Why (and when) are preferences convex? Threshold effects and uncertain quality," The B.E. Journal of Theoretical Economics, vol. 9, no. 1, 2009.

[11] A. Tasnadi, T. G. Smith, and A. S. Hanks, "Quality uncertainty as resolution of the Bertrand paradox," Pacific Economic Review, vol. 17, no. 5, pp. 687-692, 2012.

[12] K. G. Dastidar, "On the existence of pure strategy Bertrand equilibrium,” Economic Theory, vol. 5, no. 1, pp. 19-32, 1995.

[13] C. Alós-Ferrer, A. B. Ania, and K. R. Schenk-Hoppé, "An evolutionary model of Bertrand oligopoly," Games and Economic Behavior, vol. 33, no. 1, pp. 1-19, 2000.

[14] K. Abbink and J. Brandts, "24. Pricing in Bertrand competition with increasing marginal costs," Games and Economic Behavior, vol. 63, no. 1, pp. 1-31, 2008.

[15] C. Argenton and W. Müller, "Collusion in experimental Bertrand duopolies with convex costs: the role of cost asymmetry," International Journal of Industrial Organization, vol. 30, no. 6, pp. 508-517, 2012.

[16] E. H. Chamberlin, “An experimental imperfect market," Journal of Political Economy, vol. 56, no. 2, pp. 95-108, 1948.

[17] J. Apesteguia, S. Huck, and J. Oechssler, "Imitation-theory and experimental evidence," Journal of Economic Theory, vol. 136, no. 1, pp. 217-235, 2007.

[18] J. Apesteguia, S. Huck, J. Oechssler, and S. Weidenholzer, "Imitation and the evolution of Walrasian behavior: theoretically fragile but behaviorally robust," Journal of Economic Theory, vol. 145, no. 5, pp. 1603-1617, 2010.

[19] M. A. Nowak and R. M. May, "Evolutionary games and spatial chaos,” Nature, vol. 359, no. 6398, pp. 826-829, 1992.

[20] G. Szabó and C. Tőke, "Evolutionary prisoner’s dilemma game on a square lattice," Physical Review E, vol. 58, no. 1, pp. 69-73, 1998.

[21] M. Perc and A. Szolnoki, "Social diversity and promotion of cooperation in the spatial prisoner's dilemma game," Physical Review E, vol. 77, no. 1, article 011904, 2008.

[22] A. Szolnoki and G. Szabó, "Cooperation enhanced by inhomogeneous activity of teaching for evolutionary prisoner's dilemma games," Europhysics Letters, vol. 77, no. 3, article 30004, 2007.

[23] T. Killingback, M. Doebeli, and N. Knowlton, "Variable investment, the continuous prisoner's dilemma, and the origin of cooperation," Proceedings of the Royal Society of London B: Biological Sciences, vol. 266, no. 1430, pp. 1723-1728, 1999.

[24] C. Zhu, S. Sun, J. Wang, and C. Xia, "Role of population density and increasing neighborhood in the evolution of cooperation on diluted lattices," Physica A: Statistical Mechanics and its Applications, vol. 392, no. 24, pp. 6353-6360, 2013.

[25] D. J. Watts and S. H. Strogatz, "Collective dynamics of "smallworld” networks," Nature, vol. 393, no. 6684, pp. 440-442, 1998.

[26] D. J. Watts, Small Worlds: The Dynamics of Networks between Order and Randomness, Princeton University Press, 1999. 
[27] A. L. Barabási and R. Albert, "Emergence of scaling in random networks," Science, vol. 286, no. 5439, pp. 509-512, 1999.

[28] F. C. Santos, J. F. Rodrigues, and J. M. Pacheco, "Epidemic spreading and cooperation dynamics on homogeneous small-world networks," Physical Review E, vol. 72, no. 5, article 056128, 2005.

[29] Z. X. Wu, X. J. Xu, Y. Chen, and Y. H. Wang, "Spatial prisoner's dilemma game with volunteering in Newman-Watts small-world networks," Physical Review E, vol. 71, no. 3, article 037103, 2005.

[30] N. Seltzer and O. Smirnov, "Degrees of separation, social learning, and the evolution of cooperation in a small-world network," Journal of Artificial Societies and Social Simulation, vol. 18, no. 4, p. 12, 2015.

[31] F. C. Santos and J. M. Pacheco, "Scale-free networks provide a unifying framework for the emergence of cooperation," Physical Review Letters, vol. 95, no. 9, article 098104, 2005.

[32] F. C. Santos, J. M. Pacheco, and T. Lenaerts, "Evolutionary dynamics of social dilemmas in structured heterogeneous populations," Proceedings of the National Academy of Sciences, vol. 103, no. 9, pp. 3490-3494, 2006.

[33] C. Hauert and M. Doebeli, "Spatial structure often inhibits the evolution of cooperation in the snowdrift game," Nature, vol. 428, no. 6983, pp. 643-646, 2004.

[34] M. Doebeli, C. Hauert, and T. Killingback, "The evolutionary origin of cooperators and defectors," Science, vol. 306, no. 5697, pp. 859-862, 2004.

[35] K. H. Lee, C. H. Chan, P. M. Hui, and D. F. Zheng, "Cooperation in $\mathrm{N}$-person evolutionary snowdrift game in scale-free Barabási-Albert networks," Physica A: Statistical Mechanics and its Applications, vol. 387, no. 22, pp. 5602-5608, 2008.

[36] F. C. Santos, M. D. Santos, and J. M. Pacheco, "Social diversity promotes the emergence of cooperation in public goods games," Nature, vol. 454, no. 7201, pp. 213-216, 2008.

[37] X. B. Cao, W. B. Du, and Z. H. Rong, "The evolutionary public goods game on scale-free networks with heterogeneous investment," Physica A: Statistical Mechanics and its Applications, vol. 389, no. 6, pp. 1273-1280, 2010.

[38] B. Skyrms, The Stag Hunt and the Evolution of Social Structure, Cambridge University Press, 2004.

[39] S. Huck, H. T. Normann, and J. Oechssler, "Learning in Cournot oligopoly-an experiment," The Economic Journal, vol. 109, no. 454, pp. 80-95, 1999.

[40] S. Huck, H. T. Normann, and J. Oechssler, "Does information about competitors' actions increase or decrease competition in experimental oligopoly markets?," International Journal of Industrial Organization, vol. 18, no. 1, pp. 39-57, 2000.

[41] J. Yang, L. Lu, W. Xie, G. Chen, and D. Zhuang, "On competitive relationship networks: a new method for industrial competition analysis," Physica A: Statistical Mechanics and its Applications, vol. 382, no. 2, pp. 704-714, 2007.

[42] H. Rui, Y. Jian-mei, and Y. Can-zhong, "Modeling China's logistics service providers' competitive relationship based on complex networks," in 2008 International Conference on Information Management, Innovation Management and Industrial Engineering, pp. 413-418, Taipei, Taiwan, December 2008.

[43] J. Yang, W. Wang, and G. Chen, "A two-level complex network model and its application," Physica A: Statistical Mechanics and its Applications, vol. 388, no. 12, pp. 24352449, 2009.
[44] H. Gintis, Game Theory Evolving: A Problem-Centered Introduction to Modeling Strategic Behavior, Princeton University Press, 2000.

[45] J. Hofbauer and K. Sigmund, Evolutionary Games and Population Dynamics, Cambridge University Press, 1998.

[46] Z. X. Wu, J. Y. Guan, X. J. Xu, and Y. H. Wang, "Evolutionary prisoner's dilemma game on Barabási-Albert scale-free networks," Physica A: Statistical Mechanics and its Applications, vol. 379, no. 2, pp. 672-680, 2007.

[47] A. Szolnoki, M. Perc, and Z. Danku, "Towards effective payoffs in the prisoner's dilemma game on scale-free networks," Physica A: Statistical Mechanics and its Applications, vol. 387, no. 8-9, pp. 2075-2082, 2008.

[48] W. B. Du, X. B. Cao, and M. B. Hu, "The effect of asymmetric payoff mechanism on evolutionary networked prisoner's dilemma game," Physica A: Statistical Mechanics and its Applications., vol. 388, no. 24, pp. 5005-5012, 2009.

[49] T. Beom JK Ala, H. Petter, M. Petter, J. S. Chung, and M. Y. Choi, "Dynamic instabilities induced by asymmetric influence: prisoners' dilemma game in small-world networks," Physical Review E, vol. 66, article 021907, 2002.

[50] X. Thibert-Plante and L. Parrott, "Prisoner's dilemma and clusters on small-world networks," Complexity, vol. 12, no. 6, 36 pages, 2007.

[51] J. Tanimoto, "Dilemma solving by the coevolution of networks and strategy in a $2 \times 2$ game," Physical Review E, vol. 76, no. 2, article 021126, 2007.

[52] C. Hauert and G. Szabo, "Prisoner's dilemma and public goods games in different geometries: compulsory versus voluntary interactions," Complexity, vol. 8, no. 4, 38 pages, 2003.

[53] Z. Rong, X. Li, and X. Wang, "Roles of mixing patterns in cooperation on a scale-free networked game," Physical Review E, vol. 76, no. 2, article 027101, 2007.

[54] F. Xie, W. Cui, and J. Lin, "Structural heterogeneity mediates the effect of community structure on cooperation," Complexity, vol. 17, no. 4, 48 pages, 2012.

[55] G. Szabó, J. Vukov, and A. Szolnoki, "Phase diagrams for an evolutionary prisoner's dilemma game on two-dimensional lattices," Physical Review E, vol. 72, no. 4, article 047107, 2005.

[56] C. Hauert and G. Szabó, "Game theory and physics," American Journal of Physics, vol. 73, no. 5, pp. 405-414, 2005.

[57] J. P. Casasnovas, Evolutionary Games in Complex Topologies: Interplay between Structure and Dynamics, Springer Science \& Business Media, 2012.

[58] S. Nee, "Beautiful models," Nature, vol. 444, no. 7115, p. 37, 2006.

[59] N. Hanaki, A. Peterhansl, P. S. Dodds, and D. J. Watts, "Cooperation in evolving social networks," Management Science, vol. 53, no. 7, pp. 1036-1050, 2007.

[60] M. E. J. Newman, "The structure and function of complex networks," SIAM Review, vol. 45, no. 2, pp. 167-256, 2003.

[61] Z. Rong and Z.X. Wu, "Effect of the degree correlation in public goods game on scale-free networks," Europhysics Letters, vol. 87, no. 3, article 30001, 2009.

[62] X. Chen, F. Fu, and L. Wang, "Prisoner's dilemma on community networks," Physica A: Statistical Mechanics and its Applications, vol. 378, no. 2, pp. 512-518, 2007. 


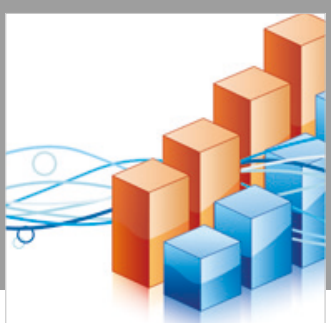

Advances in

Operations Research

\section{-n-m}
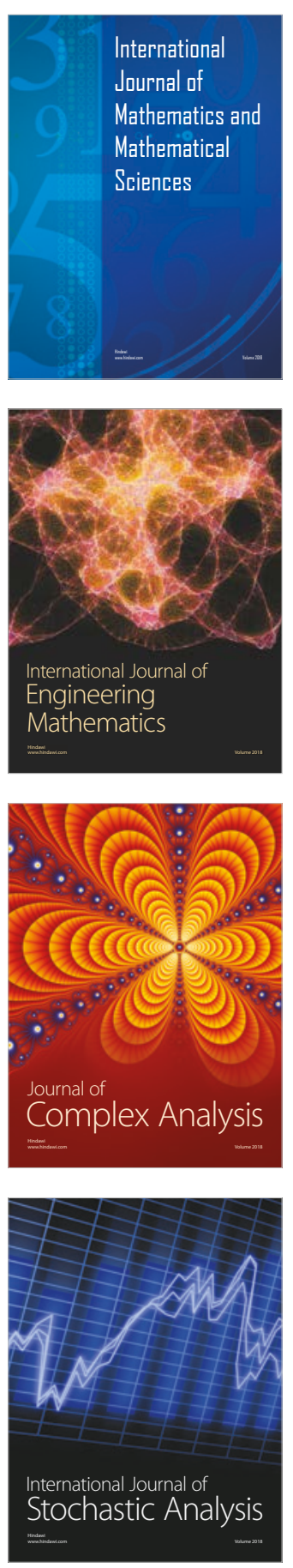
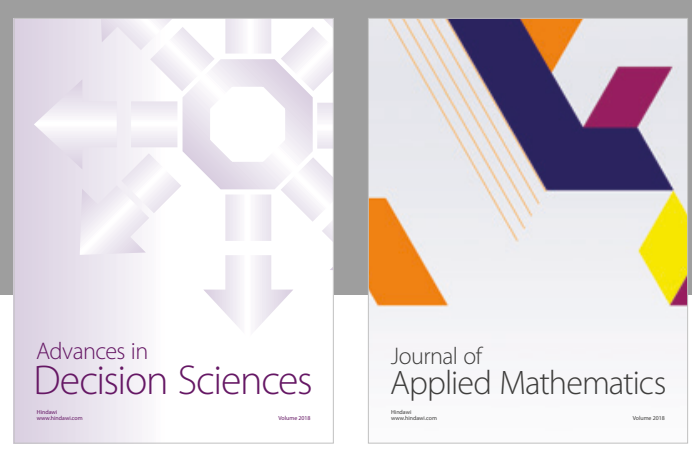

Journal of

Applied Mathematics
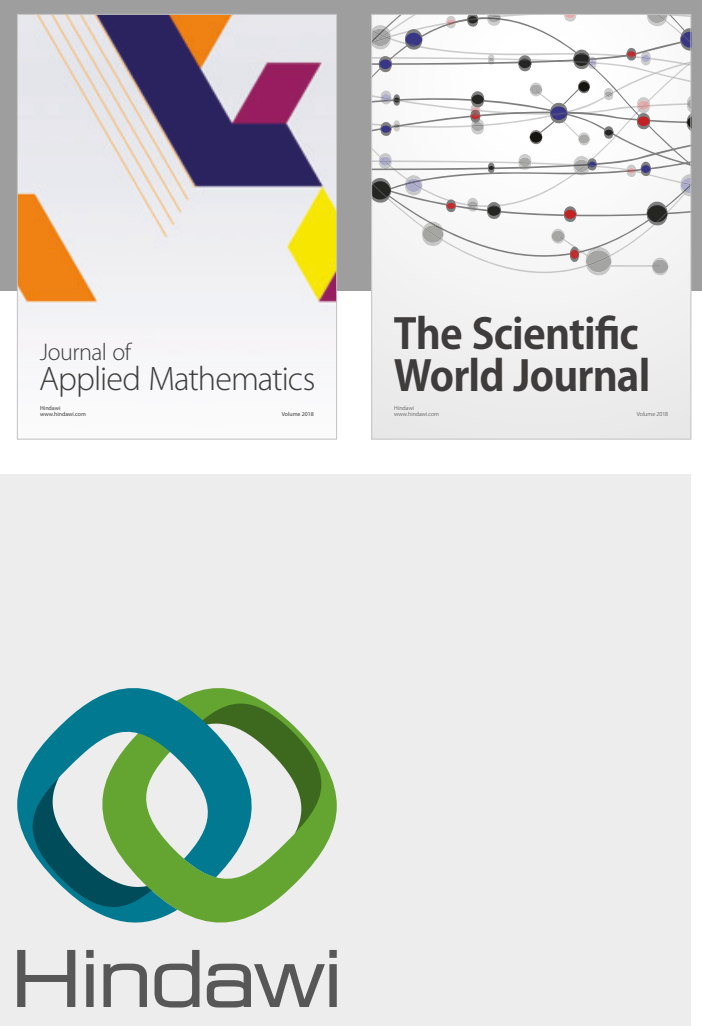

Submit your manuscripts at

www.hindawi.com

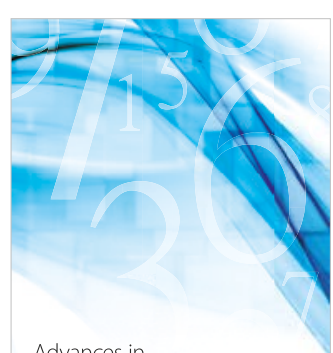

Advances in
Numerical Analysis
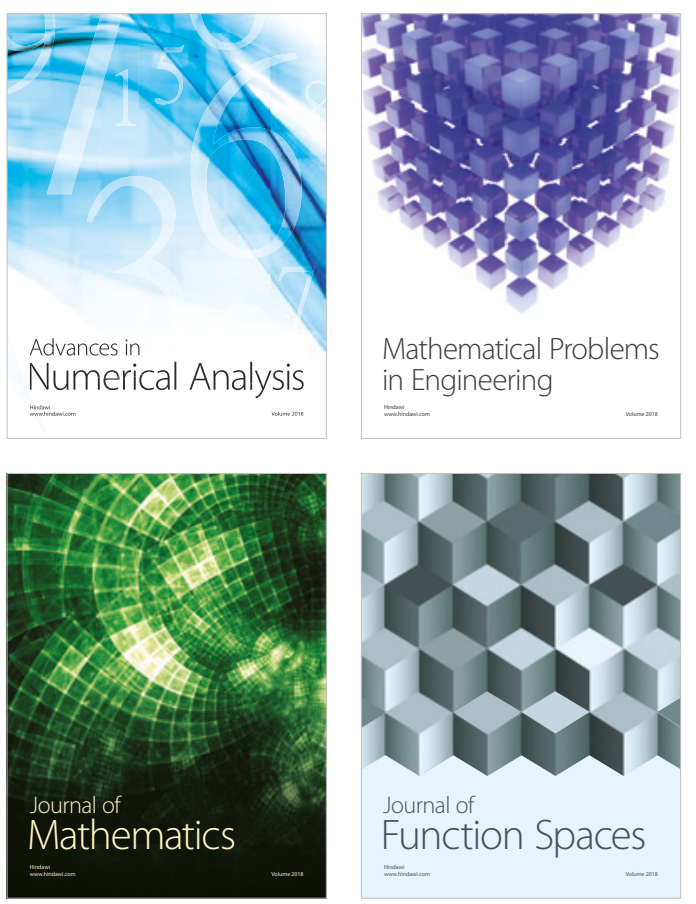

Mathematical Problems in Engineering

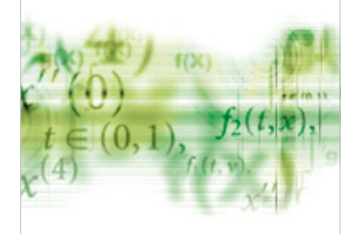

International Journal of

Differential Equations

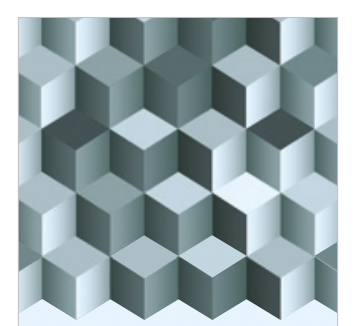

Journal of

Function Spaces
The Scientific

World Journal

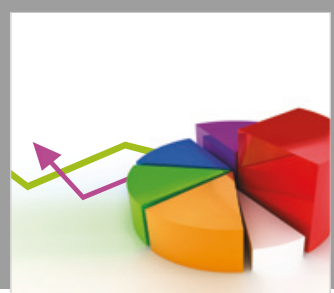

Journal of

Probability and Statistics
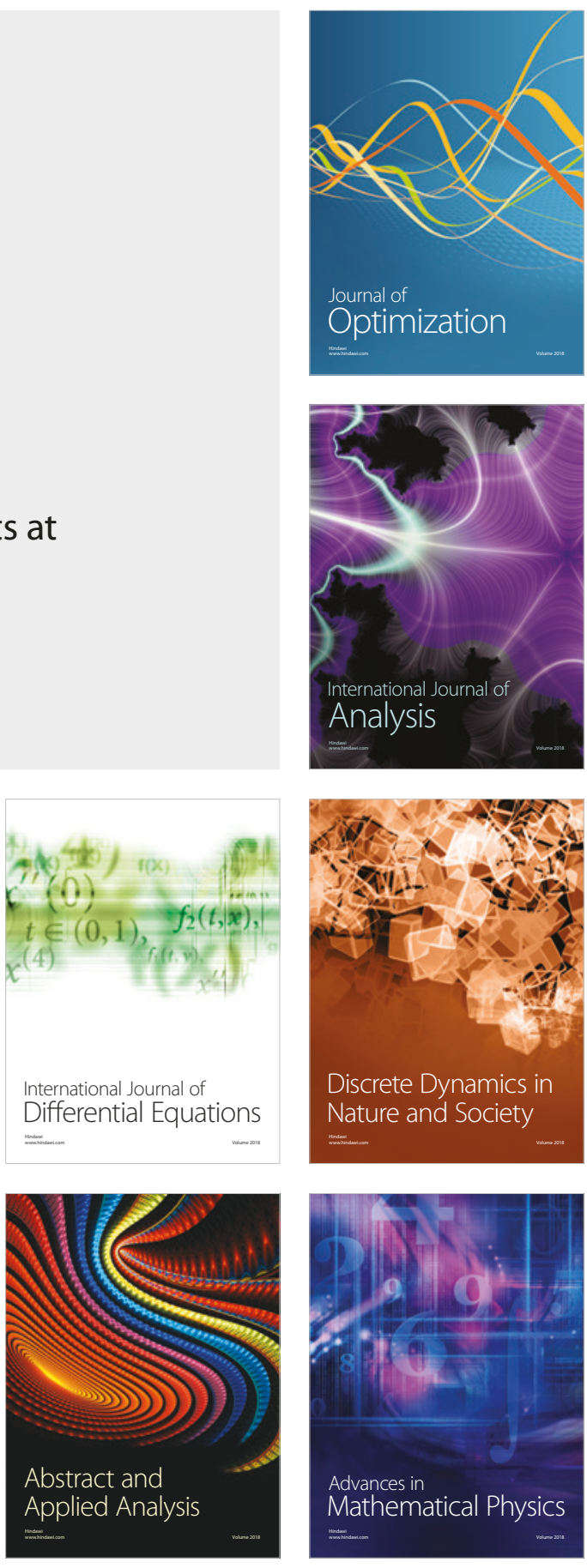